Supporting information for:

\title{
Gold-Catalyzed Addition of Carbon Nucleophiles to Propargyl Carboxylates
}

Catelijne H. M. Amijs, Verónica López-Carrillo, and Antonio M. Echavarren* Institute of Chemical Research of Catalonia (ICIQ), Av. Països Catalans 16, 43007 Tarragona, Spain

\section{Contents}

General methods

Scheme S1

General procedure for the reaction with propargyl carboxylates S2

References

Spectra

\section{General methods}

All reactions were carried out under $\mathrm{N}_{2}$ in solvents dried using a Solvent Purification System (SPS). Thin layer chromatography was carried out using TLC-aluminum sheets with $0.2 \mathrm{~mm}$ of silica gel (Merck $\mathrm{GF}_{234}$ ). Chromatography purifications were carried out using flash grade silica gel (SDS Chromatogel 60 ACC, 40-60 $\mu \mathrm{m})$. NMR spectra were recorded at $23{ }^{\circ} \mathrm{C}$ on a Bruker Avance 400 Ultrashield and Bruker Avance 500 Ultrashield apparatus. Mass spectra were recorded on a Waters LCT Premier spectrometer. Elemental analyses were performed on a LECO CHNS 932 micro-analyzer at the Universidad Complutense de Madrid. Melting points were determined using a Büchi melting point apparatus.

The following compounds were synthesized according to literature procedures: $\mathbf{1 a},{ }^{1} \mathbf{1 b},{ }^{2} \mathbf{1 c},{ }^{3} \mathbf{1 d},{ }^{4} \mathbf{1 e},{ }^{5}$ $\mathbf{1 f},{ }^{4}, \mathbf{1 2 c},{ }^{6}$ and $\mathbf{1 2 a -}-d_{2}{ }^{7}$ 
Scheme S1. Reactions of 1 a with diketone $12 \mathrm{a}$ catalyzed by $\mathrm{Ag}(\mathrm{I})$ or $\mathrm{Cu}(\mathrm{II})$ and gold-catalyzed hydration of $13 .^{a}$<smiles>[C+]#CC(OC(C)=O)c1ccccc1</smiles>

1a<smiles>O=C(CC(=O)c1ccccc1)c1ccccc1</smiles>

$12 a$

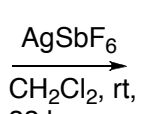

$32 \mathrm{~h}$<smiles>CC(=O)C(C(=O)c1ccccc1)C(C(C)=O)c1ccccc1</smiles>

$17(97 \%)$

$\mathrm{Cu}(\mathrm{OTf})_{2}$

$\mathrm{CH}_{2} \mathrm{Cl}_{2}$, rt, $1.5 \mathrm{~h}$

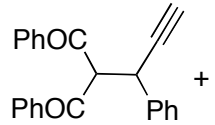

$13(20 \%)$<smiles>CC(=O)C(c1ccccc1)C(C(C)=O)c1ccccc1</smiles>

$17(79 \%)$

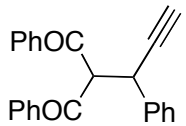

13

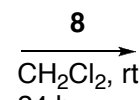

$24 \mathrm{~h}$

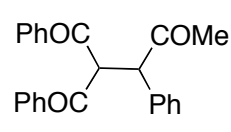

$17(79 \%)$

${ }^{a}$ Reactions with 5 mol \% catalysts.

General procedure for the reaction with propargyl carboxylates. A solution of propargyl ester and nucleophile in $\mathrm{CH}_{2} \mathrm{Cl}_{2}(1.5 \mathrm{~mL})$ was slowly added to a previously prepared mixture of the gold catalyst (2 or $5 \mathrm{~mol} \%$ rel. to the propargyl carboxylate) and, if necessary, $\mathrm{AgSbF}_{6}$ ( 2 or $5 \mathrm{~mol} \%$ rel. to the propargyl ester) and/or $\mathrm{M}(\mathrm{OTf})_{\mathrm{n}}\left(5 \mathrm{~mol} \%\right.$ rel. to the propargyl ester) in $\mathrm{CH}_{2} \mathrm{Cl}_{2}(0.5 \mathrm{~mL})$. The reaction mixture was stirred at room temperature for the time indicated in Tables 1-3 and Scheme 2-4. The mixture was filtered through silica gel with $\mathrm{CH}_{2} \mathrm{Cl}_{2}$ and the solvents were evaporated. The residue was chromatographed to give the desired product.

(Z)-5-Oxo-1,5-diphenyl-4-(phenylcarbonyl)pent-1-en-2-yl Acetate (5a).<smiles>O=C(/C=C(\CC(COc1ccccc1)c1ccccc1)c1ccccc1)OC(=O)c1ccccc1</smiles>

Compound 5a was synthesized following the general procedure (Table 1, entry 3) starting from 1a (49 $\mathrm{mg}, 0.28 \mathrm{mmol}$ ) and 12a $(94 \mathrm{mg}, 0.42 \mathrm{mmol})$. The residue was purified by chromatography ( $8: 1$ hexaneEtOAc) to give $5 \mathbf{a}(97 \mathrm{mg}, 88 \%)$ as a yellow oil. ${ }^{1} \mathrm{H}$ NMR (400 MHz, $\left.\mathrm{CDCl}_{3}\right) \delta 8.0(\mathrm{~d}, J=8.6 \mathrm{~Hz}, 4 \mathrm{H})$, $7.59(\mathrm{~m}, 2 \mathrm{H}), 7.47(\mathrm{~m}, 5 \mathrm{H}), 7.28(\mathrm{~m}, 4 \mathrm{H}), 6.11(\mathrm{~s}, 1 \mathrm{H}), 5.60(\mathrm{t}, J=6.5 \mathrm{~Hz}, 1 \mathrm{H}), 3.24(\mathrm{~d}, J=6.5 \mathrm{~Hz}, 2 \mathrm{H})$, $2.12(\mathrm{~s}, 3 \mathrm{H}) ;{ }^{13} \mathrm{C}$ NMR $\left(100 \mathrm{MHz}, \mathrm{CDCl}_{3}\right) \delta 195.27$ (C), 165.98 (C), $134.05(\mathrm{CH}), 131.22(\mathrm{C}), 129.30$ $(\mathrm{CH}), 128.71(\mathrm{CH}), 128.61(\mathrm{CH}), 128.03(\mathrm{CH}), 127.78(\mathrm{CH}), 119.41(\mathrm{CH}), 55.51(\mathrm{CH}), 34.75\left(\mathrm{CH}_{2}\right)$, $21.31\left(\mathrm{CH}_{3}\right)$; HRMS-ESI $m / z$ calcd for $\mathrm{C}_{26} \mathrm{H}_{22} \mathrm{O}_{4} \mathrm{Na}[\mathrm{M}+\mathrm{Na}]^{+} 421.1416$, found 421.1406 .

$5 \mathbf{a}-d_{2}$.<smiles>[2H]C(/C=C/c1ccccc1)C(C(=O)O)(C(=O)O)C(=O)OCc1ccccc1</smiles>

Compound $\mathbf{5 a}-\boldsymbol{d}_{\mathbf{2}}$ was synthesized following the general procedure starting from 1a $(50 \mathrm{mg}, 0.28 \mathrm{mmol})$ and 12a- $\boldsymbol{d}_{\mathbf{2}}$ (97 $\mathrm{mg}, 0.43 \mathrm{mmol}$ ) (Scheme 4$)$. The residue was purified by chromatography (8:1 hexaneEtOAc) to give $\mathbf{5 a}-\boldsymbol{d}_{\mathbf{2}}(88 \mathrm{mg}, 77 \%)$ as a yellow oil. ${ }^{1} \mathrm{H}$ NMR $\left(400 \mathrm{MHz}, \mathrm{CDCl}_{3}\right) \delta 8.0(\mathrm{~d}, J=8.6 \mathrm{~Hz}$, 
4H), $7.59(\mathrm{~m}, 2 \mathrm{H}), 7.47(\mathrm{~m}, 5 \mathrm{H}), 7.28(\mathrm{~m}, 4 \mathrm{H}), 6.11(\mathrm{~s}, 1 \mathrm{H}), 5.60(\mathrm{t}, J=6.5 \mathrm{~Hz}, 0.2 \mathrm{H}), 3.24(\mathrm{~m}, 1.6 \mathrm{H})$, $2.12(\mathrm{~s}, 3 \mathrm{H}) ;{ }^{13} \mathrm{C}$ NMR $\left(100 \mathrm{MHz}, \mathrm{CDCl}_{3}\right) \delta 195.27(\mathrm{C}), 165.98(\mathrm{C}), 134.05(\mathrm{CH}), 131.22(\mathrm{C}), 129.30$ $(\mathrm{CH}), 128.71(\mathrm{CH}), 128.61(\mathrm{CH}), 128.03(\mathrm{CH}), 127.78(\mathrm{CH}), 119.41(\mathrm{CH}), 55.06(\mathrm{CH}), 54.69(\mathrm{t}, J=19.9$ $\mathrm{Hz}, \mathrm{CD}), 34.25\left(\mathrm{CH}_{2}\right), 33.98$ (t, $\left.J=20.8 \mathrm{~Hz}, \mathrm{CHD}\right), 21.31\left(\mathrm{CH}_{3}\right)$.

(Z)-5-Oxo-1-phenyl-4-(phenylcarbonyl)hex-1-en-2-yl Acetate (5b).

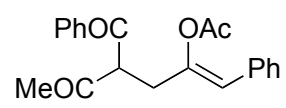

Compound $\mathbf{5 b}$ was synthesized following the general procedure (Table 2, entry 1) starting from 1a (50 $\mathrm{mg}, 0.29 \mathrm{mmol})$ and $\mathbf{1 2 b}(61 \mathrm{mg}, 0.37 \mathrm{mmol})$. The residue was purified by chromatography ( $8: 1$ hexaneEtOAc) to give $\mathbf{5 b}(55 \mathrm{mg}, 57 \%)$ as a yellow oil. ${ }^{1} \mathrm{H} \mathrm{NMR}\left(400 \mathrm{MHz}, \mathrm{CDCl}_{3}\right) \delta 8.02(\mathrm{~d}, J=7.5 \mathrm{~Hz}, 2 \mathrm{H})$, $7.62(\mathrm{~m}, 2 \mathrm{H}), 7.50(\mathrm{~m}, 2 \mathrm{H}), 7.29-7.46(\mathrm{~m}, 3 \mathrm{H}), 7.20(\mathrm{~m}, 1 \mathrm{H}), 6.06(\mathrm{~s}, 1 \mathrm{H}), 4.80(\mathrm{t}, J=6.9 \mathrm{~Hz}, 1 \mathrm{H}), 3.13$ (dd, $J=15.5,7.5 \mathrm{~Hz}, 1 \mathrm{H}), 3.06(\mathrm{dd}, J=15.5,6.9 \mathrm{~Hz}, 1 \mathrm{H}), 2.18(\mathrm{~s}, 3 \mathrm{H}) ;{ }^{13} \mathrm{C}$ NMR $\left(100 \mathrm{MHz}, \mathrm{CDCl}_{3}\right)$ 202.66 (C), 195.68 (C), 168.82 (C), 146.39 (C), 136.46 (C), 134.25 (CH), 133.73 (C), 129.00 (CH), $128.85(\mathrm{CH}), 128.41(\mathrm{CH}), 128.28(\mathrm{CH}), 127.50(\mathrm{CH}), 118.80(\mathrm{CH}), 60.82(\mathrm{CH}), 33.84\left(\mathrm{CH}_{2}\right), 28.48$ $\left(\mathrm{CH}_{3}\right), 20.97\left(\mathrm{CH}_{3}\right)$; HRMS-ESI $m / z$ calcd for $\mathrm{C}_{21} \mathrm{H}_{20} \mathrm{O}_{4} \mathrm{Na}[\mathrm{M}+\mathrm{Na}]^{+} 359.1259$, found 359.1261 .

(Z)-5-Ox0-1,5-diphenyl-4-(phenylcarbonyl)pent-1-en-2-yl Benzoate (5c).<smiles>O=C(/C=C(\O)c1ccccc1)C(Cc1ccccc1)C(=O)c1ccccc1</smiles>

Compound $\mathbf{5 c}$ was synthesized following the general procedure (Table 2, entry 2) starting from $\mathbf{1 b}$ (50 $\mathrm{mg}, 0.21 \mathrm{mmol})$ and 12a $(58 \mathrm{mg}, 0.25 \mathrm{mmol})$. The residue was purified by chromatography ( $8: 1$ hexaneEtOAc) to give $5 \mathbf{c}(90 \mathrm{mg}, 92 \%)$ as a pale yellow solid: $\mathrm{mp} 110-111^{\circ} \mathrm{C} .{ }^{1} \mathrm{H}$ NMR $\left(400 \mathrm{MHz}, \mathrm{CDCl}_{3}\right) \delta$ $8.02(\mathrm{dm}, J=8.2 \mathrm{~Hz}, 2 \mathrm{H}), 7.94(\mathrm{dm}, J=8.3 \mathrm{~Hz}, 4 \mathrm{H}), 7.63(\mathrm{~m}, 1 \mathrm{H}), 7.54-7.45(\mathrm{~m}, 4 \mathrm{H}), 7.37(\mathrm{~m}, 4 \mathrm{H})$, 7.27 (m, 2H), 7.19- $7.11(\mathrm{~m}, 3 \mathrm{H}), 6.22(\mathrm{~s}, 1 \mathrm{H}), 5.66(\mathrm{t}, J=6.4 \mathrm{~Hz}, 1 \mathrm{H}), 3.36(\mathrm{~d}, J=6.4 \mathrm{~Hz}, 2 \mathrm{H}), 2.16$ (s, $3 \mathrm{H}) ;{ }^{13} \mathrm{C}$ NMR (100 MHz, $\left.\mathrm{CDCl}_{3}\right) \delta 194.94(\mathrm{C}), 164.42(\mathrm{C}), 146.04(\mathrm{C}), 136.00(\mathrm{CH}), 133.80(\mathrm{CH})$, $133.63(\mathrm{CH}), 130.17(\mathrm{CH}), 128.89(\mathrm{CH}), 128.71(\mathrm{CH}), 128.39(\mathrm{CH}), 127.45(\mathrm{CH}), 119.64(\mathrm{CH}), 54.78$ $(\mathrm{CH}), 34.55\left(\mathrm{CH}_{2}\right)$; HRMS-ESI $\mathrm{m} / z$ calcd for $\mathrm{C}_{31} \mathrm{H}_{24} \mathrm{O}_{4} \mathrm{Na}[\mathrm{M}+\mathrm{Na}]^{+} 483.1572$, found 483.1573 .

(Z)-3-(1-(Methoxycarbonyl)-2-oxocyclohexyl)-1-phenylprop-1-en-2-yl Benzoate (5d).

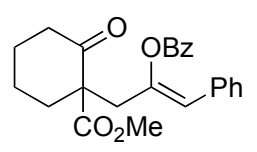

Compound $\mathbf{5 d}$ was synthesized following the general procedure (Table 2, entry 3) starting from 1a (50 $\mathrm{mg}, 0.21 \mathrm{mmol})$ and $\mathbf{1 4 a}(52 \mathrm{mg}, 0.33 \mathrm{mmol})$. The residue was purified by chromatography (8:1 hexaneEtOAc) to give 5d (41 mg, 51\%) as a white solid: $\mathrm{mp} 125-126^{\circ} \mathrm{C} .{ }^{1} \mathrm{H}$ NMR $\left(400 \mathrm{MHz}, \mathrm{CDCl}_{3}\right) \delta 8.08(\mathrm{~m}$, 2H), $7.61(\mathrm{~m}, 1 \mathrm{H}), 7.48(\mathrm{~m}, 2 \mathrm{H}), 7.35(\mathrm{~m}, 2 \mathrm{H}), 7.23-7.12(\mathrm{~m}, 3 \mathrm{H}), 6.13(\mathrm{~s}, 1 \mathrm{H}), 3.48(\mathrm{~s}, 3 \mathrm{H}), 3.29(\mathrm{~d}, J=$ 14.7, Hz, 1H), 2.80 (d, $J=14.7 \mathrm{~Hz}, 1 \mathrm{H}), 2.73(\mathrm{dm}, J=13.9 \mathrm{~Hz}, 1 \mathrm{H}), 2.47-2.31(\mathrm{~m}, 2 \mathrm{H}), 2.02(\mathrm{~m}, 1 \mathrm{H})$, 1.80-1.77 (m, 2H), $1.63(\mathrm{~m}, 2 \mathrm{H}) ;{ }^{13} \mathrm{C}$ NMR (100 MHz, $\mathrm{CDCl}_{3}$, Pendant) $\delta 206.26(\mathrm{C}), 171.42(\mathrm{C}), 164.14$ (C), $145.44(\mathrm{C}), 134.03(\mathrm{CH}), 133.60(\mathrm{C}), 130.10(\mathrm{CH}), 129.34(\mathrm{C}), 128.63(\mathrm{CH}), 128.42(\mathrm{CH}), 128.40$ 
$(\mathrm{CH}), 127.42(\mathrm{CH}), 120.78(\mathrm{CH}), 60.47(\mathrm{C}), 52.43\left(\mathrm{CH}_{3}\right), 40.92\left(\mathrm{CH}_{2}\right), 39.74\left(\mathrm{CH}_{2}\right), 35.74\left(\mathrm{CH}_{2}\right), 27.57$ $\left(\mathrm{CH}_{2}\right), 22.42\left(\mathrm{CH}_{2}\right)$; HRMS-ESI $m / z$ calcd for $\mathrm{C}_{24} \mathrm{H}_{24} \mathrm{O}_{5} \mathrm{Na}[\mathrm{M}+\mathrm{Na}]^{+} 415.1521$, found 415.1520 .

(Z)-1-(1H-Indol-3-yl)-3-methylbut-1-en-2-yl acetate (5e).<smiles>CC(=O)O/C(=C\c1c[nH]c2ccccc12)C(C)C</smiles>

Compound 5e was synthesized following the general procedure (Table 2, entry 4) starting from 1c (50 $\mathrm{mg}, 0.20 \mathrm{mmol})$ and $15(46 \mathrm{mg}, 0.20 \mathrm{mmol})$. The residue was purified by chromatography (10:1 hexaneEtOAc) to give $5 \mathbf{e}(65 \mathrm{mg}, 68 \%)$ as an off-white solid: mp $116-120^{\circ} \mathrm{C} .{ }^{1} \mathrm{H}$ NMR $\left(400 \mathrm{MHz}, \mathrm{CDCl}_{3}\right) \delta$ 8.15 (br s, 1H), 7.69 (d, $J=8.1 \mathrm{~Hz}, 1 \mathrm{H}), 7.35$ (d, $J=8.0 \mathrm{~Hz}, 1 \mathrm{H}), 7.30$ (d, $J=2.5 \mathrm{~Hz}, 1 \mathrm{H}), 7.22-7.13$ (m, $2 \mathrm{H}), 6.27(\mathrm{~s}, 1 \mathrm{H}), 2.71$ (quint, $J=6.9 \mathrm{~Hz}, 1 \mathrm{H}), 2.21(\mathrm{~s}, 3 \mathrm{H}), 1.22\left(\mathrm{~d}, J=6.9 \mathrm{~Hz}, 6 \mathrm{H} ;{ }^{13} \mathrm{C} \mathrm{NMR}(100 \mathrm{MHz}\right.$, $\left.\mathrm{CDCl}_{3}, \mathrm{DEPT}\right) \delta 168.93(\mathrm{C}), 152.83(\mathrm{C}), 135.65$ (C), $128.89(\mathrm{C}), 122.59(\mathrm{CH}), 122.56(\mathrm{CH}), 120.02$ $(\mathrm{CH}), 119.26(\mathrm{CH}), 111.20(\mathrm{CH}), 110.72(\mathrm{C}), 105.22(\mathrm{CH}), 32.82(\mathrm{CH}), 21.41\left(\mathrm{CH}_{3}\right), 20.83\left(\mathrm{CH}_{3}\right)$; HRMS-ESI $m / z$ calcd for $\mathrm{C}_{15} \mathrm{H}_{17} \mathrm{NO}_{2} \mathrm{Na}[\mathrm{M}+\mathrm{Na}]^{+}$266.1157, found 266.1152; Anal. Calcd for $\mathrm{C}_{15} \mathrm{H}_{17} \mathrm{NO}_{2} \cdot 0.5 \mathrm{H}_{2} \mathrm{O}: \mathrm{C}, 71.40 ; \mathrm{H}, 7.19$; N, 5.55; found: C, 71.84; H, 6.80; N, 5.48.

\section{5-Benzoyl-2-methyl-6-oxo-6-phenylhex-2-en-3-yl Pivalate (5f).}<smiles>CCOC(CC(C(=O)Oc1ccccc1)C(=O)c1ccccc1)=C(C)C</smiles>

Compound $\mathbf{5 f}$ was synthesized following the general procedure (Table 2, entry 5) starting from 1d (70 $\mathrm{mg}, 0.42 \mathrm{mmol})$ and 12a $(320 \mathrm{mg}, 1.43 \mathrm{mmol})$. The residue was purified by chromatography (20:1 hexane-EtOAc) to give $\mathbf{5 f}(133 \mathrm{mg}, 82 \%)$ as an off-white solid: mp $76-79^{\circ} \mathrm{C} .{ }^{1} \mathrm{H} \mathrm{NMR}\left(400 \mathrm{MHz}, \mathrm{CDCl}_{3}\right)$ $\delta 7.94(\mathrm{~d}, J=7.9 \mathrm{~Hz}, 4 \mathrm{H}), 7.56(\mathrm{t}, J=7.6 \mathrm{~Hz}, 2 \mathrm{H}), 7.44(\mathrm{t}, J=7.6 \mathrm{~Hz}, 4 \mathrm{H}), 5.43(\mathrm{t}, J=6.7 \mathrm{~Hz}, 1 \mathrm{H}), 3.09$ $(\mathrm{d}, J=6.7 \mathrm{~Hz}, 2 \mathrm{H}), 1.65(\mathrm{~s}, 3 \mathrm{H}), 1.40(\mathrm{~s}, 3 \mathrm{H}), 1.23(\mathrm{~s}, 9 \mathrm{H}) ;{ }^{13} \mathrm{C} \mathrm{NMR}\left(100 \mathrm{MHz}, \mathrm{CDCl}_{3}, \mathrm{DEPT}\right) \delta 195.59$ (C), $176.31(\mathrm{C}), 139.29(\mathrm{C}), 136.20(\mathrm{C}), 133.67(\mathrm{CH}), 128.97(\mathrm{CH}), 128.78(\mathrm{C}), 121.96(\mathrm{C}), 54.25(\mathrm{CH})$, $39.02(\mathrm{C}), 30.00\left(\mathrm{CH}_{2}\right), 27.42\left(\mathrm{CH}_{3}\right), 19.12\left(\mathrm{CH}_{3}\right), 17.58\left(\mathrm{CH}_{3}\right)$; HRMS-ESI m/z calcd for $\mathrm{C}_{25} \mathrm{H}_{29} \mathrm{O}_{4}$ $[\mathrm{M}+\mathrm{Na}]^{+}$393.2066, found 393.2083; Anal. Calcd for $\mathrm{C}_{25} \mathrm{H}_{28} \mathrm{O}_{4}$ : C, 76.50; H, 7.19; found: C, 76.10; $\mathrm{H}$, 6.98 .

\section{5-Benzoyl-2-methyl-6-oxohept-2-en-3-yl Pivalate (5g).}<smiles>CC(=O)OC(CC(C(C)=O)c1ccccc1)C(C)=O</smiles>

Compound 5g was synthesized following the general procedure (Table 2, entry 6) starting from 1d (25 $\mathrm{mg}, 0.15 \mathrm{mmol})$ and $\mathbf{1 2 b}(24 \mathrm{mg}, 0.15 \mathrm{mmol})$. The residue was purified by chromatography (20:1 hexaneEtOAc) to give $\mathbf{5 g}$ as a yellow oil $(37 \mathrm{mg}, 75 \%) .{ }^{1} \mathrm{H} \mathrm{NMR}\left(400 \mathrm{MHz}, \mathrm{CDCl}_{3}\right) \delta 7.81(\mathrm{~d}, J=7.4 \mathrm{~Hz}, 2 \mathrm{H})$, $7.60(\mathrm{t}, J=7.4 \mathrm{~Hz}, 1 \mathrm{H}), 7.48$ (t, $J=7.4 \mathrm{~Hz}, 2 \mathrm{H}), 4.63$ (t, $J=7.1 \mathrm{~Hz}, 1 \mathrm{H}), 3.00-2.90(\mathrm{~m}, 2 \mathrm{H}), 2.15(\mathrm{~s}, 3 \mathrm{H})$, $1.75(\mathrm{~s}, 3 \mathrm{H}), 1.45$ (s, 3H), $1.25(\mathrm{~s}, 9 \mathrm{H}) ;{ }^{13} \mathrm{C} \mathrm{NMR}\left(100 \mathrm{MHz}, \mathrm{CDCl}_{3}, \mathrm{DEPT}\right) \delta 203.03(\mathrm{C}), 195.99(\mathrm{C})$, 
$176.80(\mathrm{C}), 139.18(\mathrm{C}), 136.54(\mathrm{C}), 133.90(\mathrm{CH}), 128.99(\mathrm{CH}), 128.89(\mathrm{CH}), 121.85(\mathrm{C}), 60.26(\mathrm{CH})$, $39.15(\mathrm{C}), 29.35\left(\mathrm{CH}_{2}\right), 28.78\left(\mathrm{CH}_{3}\right), 27.44\left(\mathrm{CH}_{3}\right), 27.38\left(\mathrm{CH}_{3}\right), 19.09\left(\mathrm{CH}_{3}\right), 17.62\left(\mathrm{CH}_{3}\right)$; HRMS-ESI $m / z$ calcd for $\mathrm{C}_{20} \mathrm{H}_{26} \mathrm{O}_{4} \mathrm{Na}[\mathrm{M}+\mathrm{Na}]^{+} 353.1729$, found 353.1734; Anal. Calcd for $\mathrm{C}_{20} \mathrm{H}_{26} \mathrm{O}_{4}: \mathrm{C}, 72.70 ; \mathrm{H}$, 7.93; found: C, 72.68; H, 8.06.

1-(1H-Indol-3-yl)-3-methylbut-2-en-2-yl Pivalate (5h) and (Z)-1-(1H-Indol-3-yl)-3-methylbut-1-en2-yl Pivalate (5h').
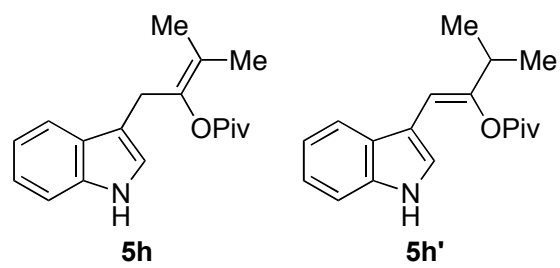

Compounds $\mathbf{5 h}$ and $\mathbf{5 h}$ ' were synthesized following the general procedure (Table 2, entry 7) starting from 1d $(50 \mathrm{mg}, 0.30 \mathrm{mmol})$ and $15(52 \mathrm{mg}, 0.45 \mathrm{mmol})$. The residue was purified by chromatography (20:1 hexane-EtOAc) to give $\mathbf{5 h}$ and $\mathbf{5 h}$ ' as a 1:1.7 mixture. Yield: 61\% (52 $\mathrm{mg}$ ), off-white solid. ${ }^{1} \mathrm{H}$ NMR (400 MHz, $\mathrm{CDCl}_{3}$ ) $\delta 8.12$ (br s, 1H, 5h'), 7.93 (br s, $1 \mathrm{H}, \mathbf{5 h}$ ), 7.66 (d, $J=7.8 \mathrm{~Hz}, 1 \mathrm{H}, \mathbf{5 h}$ '), 7.56 (d, $J=7.8 \mathrm{~Hz}$, 1H, 5h), 7.34-7.33 (m, 2H), 7.28 (d, $J=2.4 \mathrm{~Hz}, 1 \mathrm{H}, \mathbf{5 h}$ '), 7.21-7.08 (m, 4H), 6.99 (d, $J=2.2 \mathrm{~Hz}, 1 \mathrm{H}, \mathbf{5 h}$ ), 6.27 (s, 1H, 5h'), 3.75 (s, 2H, 5h), 2.70-2.60 (m, 1H, 5h'), 1.88 (s, 3H, 5h), 1.60 (s, 3H, 5h), 1.30 (s, 9H, 5h'), 1.22 (d, $J=6.7 \mathrm{~Hz}, 3 \mathrm{H}, \mathbf{5 h}$ '), 1.09 (s, 9H, 5h); ${ }^{13} \mathrm{C}$ NMR (100 MHz, CDCl 3 , DEPT) $\delta 177.04$ (C), 176.36 (C), 153.22 (C, 5h'), 141.33 (C, 5h), 136.45 (C, 5h), 135.60 (C, 5h'), 127.73 (C, 5h), 127.26 (C, 5h'), $122.62(\mathrm{CH}), 122.60(\mathrm{CH}), 122.46(\mathrm{CH}), 122.07(\mathrm{CH}), 119.94(\mathrm{CH}), 119.43(\mathrm{CH}), 119.12(\mathrm{CH})$, 118.90 (C, 5h), 112.74 (C, 5h), $111.17(\mathrm{CH}), 111.12(\mathrm{CH}), 110.52$ (C, 5i'), 105.13 (CH, 5h'), 39.30 (C, 5h'), 39.04 (C, 5h), 32.89 (CH, 5h'), $27.58\left(\mathrm{CH}_{3}, \mathbf{5 h}\right.$ '), $27.26\left(\mathrm{CH}_{3}, \mathbf{5 h}\right), 25.90\left(\mathrm{CH}_{2}, \mathbf{5 h}\right), 20.97\left(\mathrm{CH}_{3}\right.$, 5h'), $19.14\left(\mathrm{CH}_{3}, \mathbf{5 h}\right), 17.71\left(\mathrm{CH}_{3}, \mathbf{5 h}\right)$; HRMS-ESI $\mathrm{m} / z$ calcd for $\mathrm{C}_{18} \mathrm{H}_{23} \mathrm{NO}_{2} \mathrm{Na}[\mathrm{M}+\mathrm{Na}]^{+} 308.1626$, found 308.1637 .

(E)-1-Phenyl-3-(2,4,6-trimethoxyphenyl)prop-1-en-2-yl Acetate $(5 \mathrm{i})$ and 3-Phenyl-3-(2,4,6trimethoxyphenyl)prop-1-en-2-yl Acetate (6a).

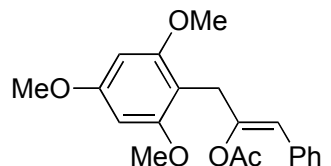

$5 \mathbf{i}$<smiles>C=C(C)C(=O)Oc1cc(OC)cc(OC)c1C(=C)OC</smiles>

$6 a$

Compound $\mathbf{5 i}$ was synthesized following the general procedure (Scheme 2) starting from 1a (50 mg, 0.28 $\mathrm{mmol})$ and 16 ( $73 \mathrm{mg}, 0.43 \mathrm{mmol})$. The residue was purified by chromatography (6:1 hexane-EtOAc) to give compound $\boldsymbol{E}-\mathbf{5 i}$ (40 $\mathrm{mg}, 40 \%$ ) as a yellow-orange oil and a 3:1 mixture of $\boldsymbol{Z}-5 \mathbf{i}$ and $\mathbf{6 a}(10 \mathrm{mg}, 10 \%)$ as a yellow oil. $\boldsymbol{E}-5 \mathbf{5}$ : ${ }^{1} \mathrm{H}$ NMR (400 MHz, $\left.\mathrm{CDCl}_{3}\right) \delta 7.20-7.30(\mathrm{~m}, 5 \mathrm{H}), 6.10(\mathrm{~s}, 2 \mathrm{H}), 5.93(\mathrm{~s}, 1 \mathrm{H}), 3.86(\mathrm{~s}$, 2H), 3.80 (s, 3H), 3.76 (s, 6H), 1.91 (s, 3H). ${ }^{13} \mathrm{C}$ NMR (100 MHz, $\left.\mathrm{CDCl}_{3}\right) \delta 169.42$ (C), 160.72 (C), $158.64(\mathrm{C}), 148.71(\mathrm{C}), 137.86(\mathrm{C}), 129.08(\mathrm{CH}), 128.31(\mathrm{CH}), 126.39(\mathrm{CH}), 109.38(\mathrm{CH}), 90.44(\mathrm{CH})$, $55.65\left(\mathrm{CH}_{3}\right), 55.26\left(\mathrm{CH}_{3}\right), 40.02\left(\mathrm{CH}_{2}\right), 21.01\left(\mathrm{CH}_{3}\right)$. HRMS-ESI calculated for $\mathrm{C}_{20} \mathrm{H}_{22} \mathrm{O}_{5} \mathrm{Na}[\mathrm{M}+\mathrm{Na}]^{+}$: 365.1365. Found: $365.1366 . \boldsymbol{Z}-5 \mathrm{i}:{ }^{1} \mathrm{H}$ NMR $\left(400 \mathrm{MHz}, \mathrm{CDCl}_{3}\right) \delta$ 7.36-7.34 (m, 2H), 7.23-7.10 (m, 3H), 
$6.10(\mathrm{~s}, 2 \mathrm{H}), 5.56(\mathrm{~m}, 1 \mathrm{H}), 4.89(\mathrm{~m}, 1 \mathrm{H}), 4.58(\mathrm{~m}, 1 \mathrm{H}), 3.77(\mathrm{~s}, 3 \mathrm{H}), 3.69(\mathrm{~s}, 6 \mathrm{H}), 1.91(\mathrm{~s}, 3 \mathrm{H}) ;{ }^{13} \mathrm{C} \mathrm{NMR}$ (100 MHz, CDCl , Pendant) $\delta 169.1$ (C), 160.8 (C), 159.2 (C), 149.6 (C), 141.3 (C), 129.2 (CH), 127.6 $(\mathrm{CH}), 126.0(\mathrm{C}), 125.8(\mathrm{CH}), 110.1(\mathrm{CH}), 91.3(\mathrm{CH}), 55.7\left(\mathrm{CH}_{3}\right), 55.6\left(\mathrm{CH}_{3}\right), 43.7\left(\mathrm{CH}_{2}\right), 21.0\left(\mathrm{CH}_{3}\right) .6 \mathrm{a}$ : ${ }^{1} \mathrm{H}$ NMR (400 MHz, $\left.\mathrm{CDCl}_{3}\right) \delta$ 7.23-7.10 (m, 5H), $6.13(\mathrm{~s}, 2 \mathrm{H}), 6.04(\mathrm{~m}, 1 \mathrm{H}), 3.80(\mathrm{~s}, 3 \mathrm{H}), 3.75(\mathrm{~s}, 6 \mathrm{H})$, 1.96 (s, 3H); ${ }^{13} \mathrm{C}$ NMR (100 MHz, $\mathrm{CDCl}_{3}$, Pendant) $\delta 169.4$ (C), 160.9 (C), 158.6 (C), 137.6 (C), 128.9 $(\mathrm{CH}), 128.0(\mathrm{CH}), 110.6(\mathrm{C}), 102.3\left(\mathrm{CH}_{2}\right), 90.6\left(\mathrm{CH}_{2}\right), 55.3\left(\mathrm{CH}_{3}\right), 55.2\left(\mathrm{CH}_{3}\right), 36.5(\mathrm{CH}), 20.8\left(\mathrm{CH}_{3}\right)$; HRMS-ESI calculated for $\mathrm{C}_{20} \mathrm{H}_{22} \mathrm{O}_{5} \mathrm{Na}[\mathrm{M}+\mathrm{Na}]^{+}, 365.1365$, found: 365.1360 .

1-(2,4,6-Trimethoxyphenyl)but-2-en-2-yl Acetate (5j).<smiles>COc1cc(C=C(C)C(=O)O[Na])c(OC)c(OC)c1</smiles>

Compound $\mathbf{5} \mathbf{j}$ was synthesized following the general procedure (Scheme 3) starting from $\mathbf{1 h}$ (51 mg, 0.45 $\mathrm{mmol})$ and 16 (113 $\mathrm{mg}, 0.67 \mathrm{mmol})$. The residue was purified by chromatography (8:1 hexane-EtOAc) to give compound $\mathbf{5 j}$ as a 2:1 mixture of isomers $Z$ and $E$. Yield: $38 \%(47 \mathrm{mg})$, pale yellow oil. ${ }^{1} \mathrm{H}$ NMR $\left(400 \mathrm{MHz}, \mathrm{CDCl}_{3}\right) \delta 6.10(\mathrm{~s}, 4 \mathrm{H}), 5.18(\mathrm{t}, J=7.8 \mathrm{~Hz}, 1 \mathrm{H}, \boldsymbol{E}), 8.09(\mathrm{t}, J=7.7 \mathrm{~Hz}, 1 \mathrm{H}), 3.78(\mathrm{~s}, 12 \mathrm{H}), 3.77$ (s, 6H), 3.28 (d, $J=7.8 \mathrm{~Hz}, 2 \mathrm{H}, \boldsymbol{E}$ ), 3.22 (d, $J=7.8 \mathrm{~Hz}, 2 \mathrm{H}, \boldsymbol{Z}$ ), 2.19 (s, 3H, Z), 2.05 (s, 3H, E), 1.99 (s, $3 \mathrm{H}, \boldsymbol{E}), 1.85(\mathrm{~s}, 3 \mathrm{H}, \boldsymbol{Z}) \cdot{ }^{13} \mathrm{C} \mathrm{NMR}\left(100 \mathrm{MHz}, \mathrm{CDCl}_{3}\right) \delta 169.7$ (C, E), $169.0(\mathrm{C}, \boldsymbol{Z}), 159.5(\mathrm{C}, \boldsymbol{E}), 159.4$ $(\mathrm{C}, \boldsymbol{Z}), 145.2(\mathrm{C}, \boldsymbol{E}), 144.1(\mathrm{C}, \boldsymbol{Z}), 116.4(\mathrm{CH}, \boldsymbol{E}), 115.8(\mathrm{CH}, \boldsymbol{Z}), 109.1(\mathrm{C}, \boldsymbol{E}), 108.9(\mathrm{C}, \boldsymbol{Z}), 90.62(\mathrm{CH}$, $\boldsymbol{Z}), 90.60(\mathrm{CH}, \boldsymbol{E}), 55.68\left(\mathrm{CH}_{3}\right), 55.65\left(\mathrm{CH}_{3}\right), 21.0\left(\mathrm{CH}_{3}, \boldsymbol{E}\right), 20.8\left(\mathrm{CH}_{3}, \boldsymbol{Z}\right), 20.2\left(\mathrm{CH}_{2}, \boldsymbol{E}\right), 19.4\left(\mathrm{CH}_{3}, \boldsymbol{Z}\right)$, $18.9\left(\mathrm{CH}_{2}, Z\right), 15.0\left(\mathrm{CH}_{3}, Z\right)$. HRMS-ESI $m / z$ calcd for $\mathrm{C}_{30} \mathrm{H}_{40} \mathrm{O}_{10} \mathrm{Na}[\mathrm{M}+\mathrm{Na}]^{+} 560.2600$, found 560.2600 .

3-(2,4,6-Trimethoxyphenyl)but-1-en-2-yl Benzoate (6b).

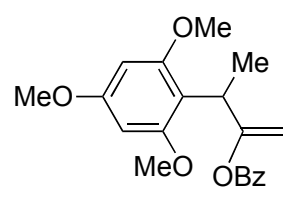

Compound $\mathbf{6 b}$ was synthesized following the general procedure (Scheme 2) starting from 1e (50 mg, 0.29 $\mathrm{mmol})$ and 16 (72 $\mathrm{mg}, 0.43 \mathrm{mmol})$. The residue was purified by chromatography (10:1 hexane-EtOAc) to give $6 \mathbf{c}$ as a colorless oil $(75 \mathrm{mg}, 76 \%) .{ }^{1} \mathrm{H}$ NMR $\left(400 \mathrm{MHz}, \mathrm{CDCl}_{3}\right): \delta 8.05(\mathrm{~d}, J=7.8 \mathrm{~Hz}, 2 \mathrm{H}), 7.53(\mathrm{t}, J$ $=7.5 \mathrm{~Hz}, 1 \mathrm{H}), 7.41(\mathrm{t}, J=7.6 \mathrm{~Hz}, 2 \mathrm{H}), 6.13(\mathrm{~s}, 2 \mathrm{H}), 5.89(\mathrm{q}, J=6.7 \mathrm{~Hz}, 1 \mathrm{H}), 5.68(\mathrm{~s}, 1 \mathrm{H}), 5.08(\mathrm{~s}, 1 \mathrm{H})$, $3.82(\mathrm{~s}, 3 \mathrm{H}), 3.70(\mathrm{~s}, 6 \mathrm{H}), 1.40(\mathrm{~d}, J=6.7 \mathrm{~Hz}, 3 \mathrm{H}) ;{ }^{13} \mathrm{C} \mathrm{NMR}\left(100 \mathrm{MHz}, \mathrm{CDCl}_{3}\right.$, DEPT) $\delta 165.94$ (C), $160.84(\mathrm{C}), 158.82(\mathrm{C}), 142.20(\mathrm{C}), 132.73(\mathrm{CH}), 131.24(\mathrm{C}), 129.78(\mathrm{CH}), 128.34(\mathrm{CH}), 116.24\left(\mathrm{CH}_{2}\right)$, $90.66(\mathrm{CH}), 73.12(\mathrm{CH}), 55.87\left(\mathrm{CH}_{3}\right), 55.48\left(\mathrm{CH}_{3}\right), 19.47\left(\mathrm{CH}_{3}\right)$; HRMS-ESI $m / z$ calcd for $\mathrm{C}_{20} \mathrm{H}_{22} \mathrm{O}_{5} \mathrm{Na}$ $[\mathrm{M}+\mathrm{Na}]^{+} 365.1365$, found 365.1372 .

(E)-3-((2-Oxocyclohexylidene)methoxy)but-1-en-2-yl Benzoate (6c). 


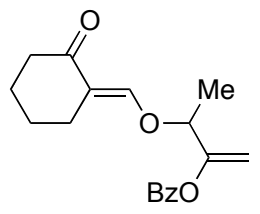

Compound $\mathbf{6 c}$ was synthesized following the general procedure (Scheme 2) starting from 1e (50 mg, 0.29 $\mathrm{mmol}$ ) and $12 \mathrm{c}(54 \mathrm{mg}, 0.43 \mathrm{mmol})$. The reaction was started with catalyst $9 / \mathrm{AgSbF}_{6}(2.5 \mathrm{~mol} \%)$ and after $3 \mathrm{~h}$ a new batch of the same catalyst $(2.5 \mathrm{~mol} \%)$ in $\mathrm{CH}_{2} \mathrm{Cl}_{2}(0.5 \mathrm{~mL})$ was added. The residue was purified by chromatography (10:1 hexane-EtOAc) to give $\mathbf{6 a}$ as a yellow oil $(52 \mathrm{mg}, 60 \%)$. ${ }^{1} \mathrm{H}$ NMR (400 MHz, $\left.\mathrm{CDCl}_{3}\right) \delta 8.05(\mathrm{~d}, J=7.7 \mathrm{~Hz}, 2 \mathrm{H}), 7.57$ (td, $\left.J=7.4 \mathrm{~Hz}, 1 \mathrm{H}\right), 7.49$ (br s, $\left.1 \mathrm{H}\right), 7.45$ (t, $J=7.6 \mathrm{~Hz}$, 2H), 5.63 (q, $J=6.5 \mathrm{~Hz}, 1 \mathrm{H}), 4.70(\mathrm{AB}, J=3.4 \mathrm{~Hz}, 1 \mathrm{H}), 4.59(\mathrm{AB}, J=3.4 \mathrm{~Hz}, 1 \mathrm{H}), 2.53(\mathrm{t}, J=6.5 \mathrm{~Hz}$, $2 \mathrm{H}), 2.40(\mathrm{t}, J=6.5 \mathrm{~Hz}, 2 \mathrm{H}), 1.86-1.80(\mathrm{~m}, 2 \mathrm{H}), 1.73-1.68(\mathrm{~m}, 2 \mathrm{H}), 1.53(\mathrm{~d}, J=6.5 \mathrm{~Hz}, 3 \mathrm{H}) ;{ }^{13} \mathrm{C} \mathrm{NMR}$ $\left(100 \mathrm{MHz}, \mathrm{CDCl}_{3}\right.$, DEPT) $\delta 200.68(\mathrm{C}), 165.49(\mathrm{C}), 159.82(\mathrm{C}), 148.90(\mathrm{CH}), 133.16(\mathrm{CH}), 130.04(\mathrm{C})$, $129.70(\mathrm{CH}), 128.42(\mathrm{CH}), 119.70(\mathrm{C}), 91.34\left(\mathrm{CH}_{2}\right), 69.67(\mathrm{CH}), 39.96\left(\mathrm{CH}_{2}\right), 23.54\left(\mathrm{CH}_{2}\right), 23.06\left(\mathrm{CH}_{2}\right)$, $22.65\left(\mathrm{CH}_{2}\right), 18.22\left(\mathrm{CH}_{3}\right)$; HRMS-ESI $m / z$ calcd for $\mathrm{C}_{18} \mathrm{H}_{20} \mathrm{O}_{4} \mathrm{Na}[\mathrm{M}+\mathrm{Na}]^{+} 323.1259$, found 323.1249; Anal. Calcd for $\mathrm{C}_{18} \mathrm{H}_{20} \mathrm{O}_{4} \cdot 2 \mathrm{H}_{2} \mathrm{O}: \mathrm{C}, 64.27$; H, 7.19; found: C, 63.74; H, 7.21.

\section{(E)-4-(Methoxycarbonyl)-3-methyl-5-oxonon-1-enyl Benzoate (7a).}

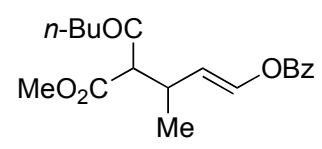

Compound 7a was synthesized following the general procedure (Table 3, entry 1) starting from 1e (15 $\mathrm{mg}, 0.09 \mathrm{mmol})$ and 14b $(21 \mathrm{mg}, 0.13 \mathrm{mmol})$. The residue was purified by chromatography (100:1 hexane-EtOAc) to give $7 \mathbf{a}$ as a 1.2:1mixture of diastereomers as a colorless oil (17 mg, 58\%). ${ }^{1} \mathrm{H} \mathrm{NMR}$ $\left(400 \mathrm{MHz}, \mathrm{CDCl}_{3}\right) \delta 8.08-8.05(\mathrm{~m}, 4 \mathrm{H}), 7.59$ (dt, $\left.J=7.5,1.2 \mathrm{~Hz}, 2 \mathrm{H}\right), 7.47-7.43$ (m, 4H), 7.39 (dd, $J=$ 3.4, $0.7 \mathrm{~Hz}, 1 \mathrm{H}), 7.36(\mathrm{dd}, J=3.4,0.8 \mathrm{~Hz}, 1 \mathrm{H}), 5.56(\mathrm{dd}, J=12.5,9.2 \mathrm{~Hz}, 1 \mathrm{H}), 5.10$ (dd, $J=12.5,9.1$ Hz, 1H), 3.73 (s, 3H), 3.71 (s, 3H), 3.46 (d, $J=9.3 \mathrm{~Hz}, 2 \mathrm{H}), 3.14-3.05$ (m, 2H), 2.65-2.41 (m, 4H), 1.59$1.50(\mathrm{~m}, 4 \mathrm{H}), 1.33-1.25(\mathrm{~m}, 4 \mathrm{H}), 1.14(\mathrm{~d}, J=6.8 \mathrm{~Hz}, 3 \mathrm{H}), 1.09(\mathrm{~d}, J=6.7 \mathrm{~Hz}, 3 \mathrm{H}), 0.90(\mathrm{t}, J=7.4 \mathrm{~Hz}$, $3 \mathrm{H}), 0.87(\mathrm{t}, J=7.4 \mathrm{~Hz}, 3 \mathrm{H}) ;{ }^{13} \mathrm{C} \mathrm{NMR}\left(100 \mathrm{MHz}, \mathrm{CDCl}_{3}, \mathrm{DEPT}\right) \delta 204.36$ (C), $204.22(\mathrm{C}), 169.12(\mathrm{C})$, $168.95(\mathrm{C}), 163.81(\mathrm{C}), 163.74(\mathrm{C}), 136.87(\mathrm{CH}), 136.84(\mathrm{CH}), 133.70(\mathrm{CH}), 130.10(\mathrm{CH}), 129.16(\mathrm{C})$, $129.13(\mathrm{C}), 128.67(\mathrm{CH}), 116.99(\mathrm{CH}), 116.87(\mathrm{CH}), 65.51(\mathrm{CH}), 65.22(\mathrm{CH}), 52.56\left(\mathrm{CH}_{3}\right), 42.84\left(\mathrm{CH}_{2}\right)$, $42.81\left(\mathrm{CH}_{2}\right), 33.01(\mathrm{CH}), 32.96(\mathrm{CH}), 25.58\left(\mathrm{CH}_{2}\right), 25.46\left(\mathrm{CH}_{2}\right), 22.29\left(\mathrm{CH}_{2}\right), 22.24\left(\mathrm{CH}_{2}\right), 18.98\left(\mathrm{CH}_{3}\right)$, $13.96\left(\mathrm{CH}_{3}\right), 13.95\left(\mathrm{CH}_{3}\right)$; HRMS-ESI $m / z$ calcd for $\mathrm{C}_{19} \mathrm{H}_{24} \mathrm{O}_{5} \mathrm{Na}[\mathrm{M}+\mathrm{Na}]^{+}$355.1521, found 355.1510; Anal. Calcd for $\mathrm{C}_{19} \mathrm{H}_{24} \mathrm{O}_{5}$ : C, 68.66; H, 7.28; found: C, 68.18; H, 7.58.

(E)-4-(Ethoxycarbonyl)-4-ethyl-3-methyl-5-oxohex-1-enyl Benzoate (7b).

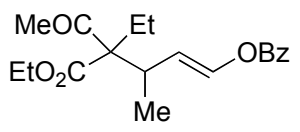

Compound $\mathbf{7 b}$ was synthesized following the general procedure (Table 3, entry 2) starting from 1e (15 $\mathrm{mg}, 0.09 \mathrm{mmol})$ and $14 \mathrm{c}(23 \mathrm{mg}, 0.13 \mathrm{mmol})$. The residue was purified by chromatography (20:1 hexaneEtOAc) to give $7 \mathbf{b}$ as a 1.1:1 mixture of diastereomers as a colorless oil (14 mg, 49\%). ${ }^{1} \mathrm{H}$ NMR (400 
$\left.\mathrm{MHz}, \mathrm{CDCl}_{3}\right) \delta 8.10-8.06(\mathrm{~m}, 4 \mathrm{H}), 7.61-7.57(\mathrm{~m}, 2 \mathrm{H}), 7.48-7.44(\mathrm{~m}, 2 \mathrm{H}), 7.39(\mathrm{dd}, J=12.5,0.6 \mathrm{~Hz}, 1 \mathrm{H})$, $7.34(\mathrm{dd}, J=12.5,0.9 \mathrm{~Hz}, 1 \mathrm{H}), 5.66(\mathrm{dd}, J=12.5,8.9 \mathrm{~Hz}, 1 \mathrm{H}), 5.57$ (dd, $J=12.5,10.0 \mathrm{~Hz}, 1 \mathrm{H}), 4.29$ $4.21(\mathrm{~m}, 4 \mathrm{H}), 3.07-2.97(\mathrm{~m}, 2 \mathrm{H}), 2.19(\mathrm{~s}, 3 \mathrm{H}), 2.18(\mathrm{~s}, 3 \mathrm{H}), 2.04-1.85(\mathrm{~m}, 4 \mathrm{H}), 1.32(\mathrm{t}, J=7.1 \mathrm{~Hz}, 3 \mathrm{H})$, $1.31(\mathrm{t}, J=7.1 \mathrm{~Hz}, 3 \mathrm{H}), 1.14(\mathrm{~d}, J=7.1 \mathrm{~Hz}, 3 \mathrm{H}), 1.07$ (d, $J=7.1 \mathrm{~Hz}, 3 \mathrm{H}), 0.85$ (t, $J=7.6 \mathrm{~Hz}, 3 \mathrm{H}), 0.80$ $(\mathrm{t}, J=7.6 \mathrm{~Hz}, 3 \mathrm{H}) ;{ }^{13} \mathrm{C}$ NMR (100 MHz, $\mathrm{CDCl}_{3}$, DEPT) $\delta 205.56$ (C), 205.09 (C), 171.74 (C), 163.84 (C), $136.73(\mathrm{CH}), 136.67(\mathrm{CH}), 133.66(\mathrm{CH}), 133.60(\mathrm{CH}), 130.09(\mathrm{CH}), 130.08(\mathrm{CH}), 129.27(\mathrm{C})$, $129.20(\mathrm{C}), 128.65(\mathrm{CH}), 128.63(\mathrm{CH}), 116.95(\mathrm{CH}), 116.34(\mathrm{CH}), 67.21(\mathrm{C}), 66.70(\mathrm{C}), 61.17\left(\mathrm{CH}_{2}\right)$, $35.40(\mathrm{CH}), 34.71(\mathrm{CH}), 29.36\left(\mathrm{CH}_{3}\right), 26.27\left(\mathrm{CH}_{2}\right), 26.07\left(\mathrm{CH}_{2}\right), 17.82\left(\mathrm{CH}_{3}\right), 16.51\left(\mathrm{CH}_{3}\right), 14.37\left(\mathrm{CH}_{3}\right)$, $14.32\left(\mathrm{CH}_{3}\right), 8.37\left(\mathrm{CH}_{3}\right)$; HRMS-ESI $\mathrm{m} / z$ calcd for $\mathrm{C}_{19} \mathrm{H}_{24} \mathrm{O}_{5} \mathrm{Na}[\mathrm{M}+\mathrm{Na}]^{+}$355.1521, found 355.1523; Anal. Calcd for $\mathrm{C}_{19} \mathrm{H}_{24} \mathrm{O}_{5}$ : C, 68.66; H, 7.28; found: C, 68.89; H, 7.28.

(E)-4-Acetyl-3-methyl-5-oxohex-1-enyl Benzoate (7c).

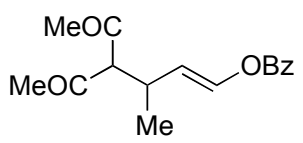

Compound 7c was synthesized following the general procedure (Table 3, entry 3) starting from 1e (50 $\mathrm{mg}, 0.29 \mathrm{mmol})$ and $\mathbf{1 2 d}(144 \mathrm{mg}, 1.43 \mathrm{mmol})$. The residue was purified by chromatography $(10: 1$ hexane-EtOAc) to give $7 \mathrm{c}(36 \mathrm{mg}, 46 \%)$ as a white solid: $\mathrm{mp} 79-81^{\circ} \mathrm{C} .{ }^{1} \mathrm{H}$ NMR $\left(400 \mathrm{MHz}, \mathrm{CDCl}_{3}\right) \delta$ $8.06(\mathrm{~d}, J=8.0 \mathrm{~Hz}, 2 \mathrm{H}), 7.59$ (t, $J=7.7 \mathrm{~Hz}, 1 \mathrm{H}), 7.46$ (t, $J=7.7 \mathrm{~Hz}, 2 \mathrm{H}), 7.40$ (d, $J=12.6 \mathrm{~Hz}, 1 \mathrm{H}), 5.45$ (dd, $J=12.3,9.1 \mathrm{~Hz}, 1 \mathrm{H}), 3.64(\mathrm{~d}, J=10.4 \mathrm{~Hz}, 3 \mathrm{H}), 3.19-3.09(\mathrm{~m}, 1 \mathrm{H}), 2.22(\mathrm{~s}, 3 \mathrm{H}), 2.17(\mathrm{~s}, 3 \mathrm{H}), 1.09$ $(\mathrm{d}, J=6.8 \mathrm{~Hz}, 3 \mathrm{H}) ;{ }^{13} \mathrm{C} \mathrm{NMR}\left(100 \mathrm{MHz}, \mathrm{CDCl}_{3}, \mathrm{DEPT}\right) \delta 203.47$ (C), 203.41 (C), $163.78(\mathrm{C}), 136.90$ $(\mathrm{CH}), 133.80(\mathrm{CH}), 130.14(\mathrm{CH}), 128.71(\mathrm{CH}), 116.66(\mathrm{CH}), 75.92(\mathrm{CH}), 33.38(\mathrm{CH}), 30.18\left(\mathrm{CH}_{3}\right)$, $29.72\left(\mathrm{CH}_{3}\right), 19.29\left(\mathrm{CH}_{3}\right)$; HRMS-ESI $\mathrm{m} / z$ calcd for $\mathrm{C}_{16} \mathrm{H}_{18} \mathrm{O}_{4} \mathrm{Na}[\mathrm{M}+\mathrm{Na}]^{+}$297.1103, found 297.1092; Anal. Calcd for $\mathrm{C}_{16} \mathrm{H}_{18} \mathrm{O}_{4}$ : C, 70.06; H, 6.61; found: C, 69.55; H, 6.46.

(E)-4-Benzoyl-3-methyl-5-oxo-5-phenylpent-1-enyl Pivalate (7d) and (E)-2-Benzoyl-1-0xo-1phenylhex-4-en-3-yl Pivalate (7d').
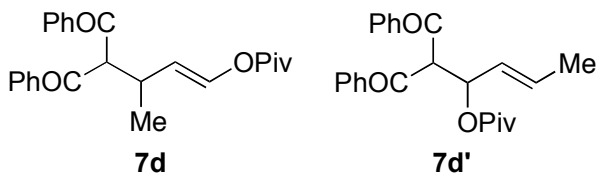

Compounds 7d and 7d' were synthesized following the general procedure (Table 3, entry 4) starting from 1f $(50 \mathrm{mg}, 0.32 \mathrm{mmol})$ and $\mathbf{1 2 a}(145 \mathrm{mg}, 0.65 \mathrm{mmol})$. The residue was purified by chromatography $(5: 1$ hexane-EtOAc) to give 7c and 7c' as a 1.1:1 mixture. Yield: 78\% (95 mg), yellow oil. ${ }^{1} \mathrm{H}$ NMR (400 $\left.\mathrm{MHz}, \mathrm{CDCl}_{3}\right) \delta 8.04\left(\mathrm{~d}, J=7.4 \mathrm{~Hz}, 2 \mathrm{H}, 7 \mathbf{d}^{\prime}\right), 7.98(\mathrm{~d}, J=7.7 \mathrm{~Hz}, 2 \mathrm{H}, 7 \mathbf{d}), 7.94(\mathrm{~d}, J=7.7 \mathrm{~Hz}, 4 \mathrm{H}), 7.60-$ $7.50(\mathrm{~m}, 5 \mathrm{H}), 7.49-7.40(\mathrm{~m}, 9 \mathrm{H}), 7.07$ (d, $J=12.6 \mathrm{~Hz}, 1 \mathrm{H}, 7 \mathbf{d}), 6.20-6.16\left(\mathrm{~m}, 1 \mathrm{H}, 7 \mathbf{d}^{\prime}\right), 5.78(\mathrm{dq}, J=$ 15.4, $6.6 \mathrm{~Hz}, 1 \mathrm{H}, 7 \mathbf{d}^{\prime}$ ), 5.69 (d, $J=9.1 \mathrm{~Hz}, 1 \mathrm{H}, 7 \mathbf{d}^{\prime}$ ), 5.55 (ddd, $\left.J=15.4,7.1,1.6, \mathrm{~Hz}, 1 \mathrm{H}, 7 \mathbf{d}^{\prime}\right), 5.47$ (dd, $J=12.5,8.7, \mathrm{~Hz}, 1 \mathrm{H}, 7 \mathbf{d}), 5.23$ (d, $J=8.8 \mathrm{~Hz}, 1 \mathrm{H}, 7 \mathbf{d}), 3.49-3.39(\mathrm{~m}, 1 \mathrm{H}, 7 \mathbf{d}), 1.59$ (dd, $J=6.4,1.2 \mathrm{~Hz}$, $3 \mathrm{H}, 7$ 'd), 1.17 (d, $J=7.01 \mathrm{~Hz}, 3 \mathrm{H}, 7 \mathbf{d}), 1.15$ (s, 9H), 0.97 (s, 9H); ${ }^{13} \mathrm{C}$ NMR (100 MHz, CDCl 3 , DEPT) $\delta$ 195.07 (C, 7d), 195.04 (C, 7d), 192.89 (C, 7d'), 192.70 (C, 7d'), 176.97 (C, 7d'), 175.65 (C, 7d), 137.05 (C), $136.95(\mathrm{CH}, 7 d), 136.76(\mathrm{C}), 133.85(\mathrm{CH}), 133.80(\mathrm{CH}), 133.77(\mathrm{CH}), 133.58(\mathrm{CH}), 131.17(\mathrm{CH}$, 
7d'), $129.08(\mathrm{CH}), 129.05(\mathrm{CH}), 129.01(\mathrm{CH}), 128.98(\mathrm{CH}), 128.88(\mathrm{CH}), 128.85(\mathrm{CH}), 127.36(\mathrm{CH}$, 7' '), $116.90(\mathrm{CH}, 7 \mathbf{d}), 74.08\left(\mathrm{CH}, 7 \mathbf{d}^{\prime}\right), 63.31(\mathrm{CH}, 7 \mathbf{d}), 61.44\left(\mathrm{CH}, 7 \mathbf{d}^{\prime}\right), 38.82(\mathrm{C}), 38.74(\mathrm{C}), 34.13$ $(\mathrm{CH}, 7 \mathbf{d}), 27.05\left(\mathrm{CH}_{3}\right), 26.99\left(\mathrm{CH}_{3}\right), 19.08\left(\mathrm{CH}_{3}, 7 \mathbf{d}\right), 17.95\left(\mathrm{CH}_{3}, 7 \mathbf{d}\right)$; HRMS-ESI $m / z$ calcd for $\mathrm{C}_{24} \mathrm{H}_{26} \mathrm{O}_{4} \mathrm{Na}[\mathrm{M}+\mathrm{Na}]^{+} 401.1729$, found 401.1728 .

Methyl (E)-2-(4-(2,2-Dimethylpropanoyloxy)but-3-en-2-yl)-3-oxoheptanoate (7e).<smiles>CC(=O)OCC(=O)OCC(C)C(C)C=CO[Na]</smiles>

Compound 7e was synthesized following the general procedure (Table 3, entry 5) starting from 1f (15 $\mathrm{mg}, 0.10 \mathrm{mmol})$ and $\mathbf{1 4 b}(24 \mathrm{mg}, 0.15 \mathrm{mmol})$. The residue was purified by chromatography (50:1 hexaneEtOAc) to give 7e as a 1:1.05 mixture of diastereomers as a colorless oil (16 mg, 53\%). ${ }^{1} \mathrm{H}$ NMR (400 $\left.\mathrm{MHz}, \mathrm{CDCl}_{3}\right) \delta 7.11(\mathrm{~d}, J=12.4 \mathrm{~Hz}, 2 \mathrm{H}), 5.37(\mathrm{dd}, J=12.4,9.0 \mathrm{~Hz}, 1 \mathrm{H}), 5.31(\mathrm{dd}, J=12.4,9.0 \mathrm{~Hz}, 1 \mathrm{H})$, 3.72 (s, 3H), 3.70 (s, 3H), 3.41 (dd, $J=9.4,0.8 \mathrm{~Hz}, 2 \mathrm{H}), 3.05-2.96$ (m, 2H), 2.59-2.39 (m, 4H), 1.59-1.49 (m, 4H), 1.34-1.23 (m, 4H), $1.22(\mathrm{~s}, 9 \mathrm{H}), 1.21(\mathrm{~s}, 9 \mathrm{H}), 1.10(\mathrm{~d}, J=6.8 \mathrm{~Hz}, 3 \mathrm{H}), 1.05(\mathrm{~d}, J=6.6 \mathrm{~Hz}, 3 \mathrm{H})$, 0.88 (q, $J=7.2 \mathrm{~Hz}, 6 \mathrm{H}) ;{ }^{13} \mathrm{C}$ NMR $\left(100 \mathrm{MHz}, \mathrm{CDCl}_{3}, \mathrm{DEPT}\right) \delta 204.45$ (C), 204.31 (C), 175.78 (C), $175.66(\mathrm{C}), 169.12(\mathrm{C}), 168.98(\mathrm{C}), 137.07(\mathrm{CH}), 137.05(\mathrm{CH}), 116.13(\mathrm{CH}), 115.92(\mathrm{CH}), 65.49(\mathrm{CH})$, $65.25(\mathrm{CH}), 52.52\left(\mathrm{CH}_{3}\right), 52.50\left(\mathrm{CH}_{3}\right), 42.82\left(\mathrm{CH}_{2}\right), 42.80\left(\mathrm{CH}_{2}\right), 38.81(\mathrm{C}), 33.00(\mathrm{CH}), 32.91(\mathrm{CH})$, $27.10\left(\mathrm{CH}_{3}\right), 27.08\left(\mathrm{CH}_{3}\right), 25.57\left(\mathrm{CH}_{2}\right), 25.44\left(\mathrm{CH}_{2}\right), 22.28\left(\mathrm{CH}_{2}\right), 22.24\left(\mathrm{CH}_{2}\right), 19.04\left(\mathrm{CH}_{3}\right), 18.93$ $\left(\mathrm{CH}_{3}\right), 14.29\left(\mathrm{CH}_{3}\right), 13.97\left(\mathrm{CH}_{3}\right)$; HRMS-ESI $\mathrm{m} / z$ calcd for $\mathrm{C}_{17} \mathrm{H}_{28} \mathrm{O}_{5} \mathrm{Na}[\mathrm{M}+\mathrm{Na}]^{+} 335.1834$, found 335.1837; Anal. Calcd for $\mathrm{C}_{17} \mathrm{H}_{28} \mathrm{O}_{5}$ : C, 65.36; H, 9.03; found: C, 65.52; H, 8.83.

Ethyl (E)-2-benzoyl-3-methyl-5-(pivaloyloxy)pent-4-enoate (7f).<smiles>CCOC(=O)C(C=COC)C(Oc1ccccc1)[N+](=O)[O-]</smiles>

Compound 7f was synthesized following the general procedure (Table 3, entry 6) starting from $\mathbf{1 f}$ (15 mg, $0.10 \mathrm{mmol})$ and $14 \mathrm{~d}(28 \mathrm{mg}, 0.15 \mathrm{mmol})$. The residue was purified by chromatography (50:1 hexaneEtOAc) to give a mixture of diastereomers (1.1:1) of $7 \mathbf{f}$ as a colorless oil $(20 \mathrm{mg}, 55 \%),{ }^{1} \mathrm{H}$ NMR (400 $\left.\mathrm{MHz}, \mathrm{CDCl}_{3}\right) \delta 8.02(\mathrm{dd}, J=7.2,1.3 \mathrm{~Hz}, 1 \mathrm{H}), 7.97(\mathrm{dd}, J=7.2,1.3 \mathrm{~Hz}, 1 \mathrm{H}), 7.58(\mathrm{dt}, J=7.2,1.3 \mathrm{~Hz}$, 2H), 7.50-7.45 (m, 4H), $7.16(\mathrm{dd}, J=10.7,0.9 \mathrm{~Hz}, 1 \mathrm{H}), 7.13(\mathrm{dd}, J=10.7,0.9 \mathrm{~Hz}, 1 \mathrm{H}), 5.47$ (dd, $J=$ $12.3,9.2, \mathrm{~Hz}, 1 \mathrm{H}), 5.30(\mathrm{dd}, J=12.5,8.6 \mathrm{~Hz}, 1 \mathrm{H}), 4.27-4.23(\mathrm{~m}, 2 \mathrm{H}), 4.18-4.08(\mathrm{~m}, 2 \mathrm{H}), 1.22(\mathrm{~s}, 9 \mathrm{H})$, 1.20-1.17 (m, 9H), 1.17 (s, 9H), 1.09 (d, $J=6.6 \mathrm{~Hz}, 3 \mathrm{H}) ;{ }^{13} \mathrm{C}$ NMR (100 MHz, $\mathrm{CDCl}_{3}$, DEPT) $\delta 193.97$ (C), $193.77(\mathrm{C}), 168.67(\mathrm{C}), 137.13(\mathrm{CH}), 137.01(\mathrm{CH}), 136.86(\mathrm{C}), 133.81(\mathrm{CH}), 133.63(\mathrm{CH}), 128.96$ $(\mathrm{CH}), 128.89(\mathrm{CH}), 128.79(\mathrm{CH}), 128.73(\mathrm{CH}), 116.55(\mathrm{CH}), 116.12(\mathrm{CH}), 61.66\left(\mathrm{CH}_{2}\right), 60.85(\mathrm{CH})$, $60.40(\mathrm{CH}), 38.38(\mathrm{C}), 33.30(\mathrm{CH}), 32.93(\mathrm{CH}), 27.10\left(\mathrm{CH}_{3}\right), 27.06\left(\mathrm{CH}_{3}\right), 19.44\left(\mathrm{CH}_{3}\right), 18.44\left(\mathrm{CH}_{3}\right)$, $14.20\left(\mathrm{CH}_{3}\right)$; HRMS-ESI $m / z$ calcd for $\mathrm{C}_{20} \mathrm{H}_{26} \mathrm{O}_{5} \mathrm{Na}[\mathrm{M}+\mathrm{Na}]^{+} 369.1678$, found 369.1680; Anal. Calcd for $\mathrm{C}_{20} \mathrm{H}_{26} \mathrm{O}_{5}$ : C, 69.34; H, 7.56; found: C, 69.52; H, 7.53.

(Z)-5-Oxo-5-phenyl-4-(phenylcarbonyl)pent-1-enyl Acetate (7g).<smiles>CC(=O)O/C=C/CC(Cc1ccccc1)C(=O)c1ccccc1</smiles> 
Compound $7 \mathbf{g}$ was synthesized following the general procedure (Table 3, entry 7) starting from $\mathbf{1 g}(0.054$ $\mathrm{mL}, 0.48 \mathrm{mmol})$ and $12 \mathrm{a}(154 \mathrm{mg}, 0.67 \mathrm{mmol})$. The residue was purified by chromatography (10:1 hexane-EtOAc) to give $7 \mathrm{~g}(136 \mathrm{mg}, 87 \%)$ as a white solid: $\mathrm{mp} 160-162{ }^{\circ} \mathrm{C} .{ }^{1} \mathrm{H} \mathrm{NMR}\left(400 \mathrm{MHz}, \mathrm{CDCl}_{3}\right) \delta$ $7.94(\mathrm{~m}, 4 \mathrm{H}), 7.57(\mathrm{~m}, 2 \mathrm{H}), 7.45(\mathrm{~m}, 4 \mathrm{H}), 7.15(\mathrm{dm}, J=12.5 \mathrm{~Hz}, 1 \mathrm{H}), 5.50(\mathrm{dt}, J=12.5,7.9 \mathrm{~Hz}, 1 \mathrm{H})$, $5.23(\mathrm{t}, J=6.6 \mathrm{~Hz}, 1 \mathrm{H}), 2.80(\mathrm{~m}, 2 \mathrm{H}), 2.07(\mathrm{~s}, 3 \mathrm{H}) ;{ }^{13} \mathrm{C} \mathrm{NMR}\left(100 \mathrm{MHz}, \mathrm{CDCl}_{3}\right) \delta 195.68(\mathrm{C}), 168.09$ (C), $137.56(\mathrm{CH}), 135.74(\mathrm{C}), 133.88(\mathrm{CH}), 128.87(\mathrm{CH}), 128.50(\mathrm{CH}), 111.36(\mathrm{CH}), 57.29(\mathrm{CH}), 27.40$ $\left(\mathrm{CH}_{2}\right), 20.54\left(\mathrm{CH}_{3}\right)$; HRMS-ESI $m / z$ calcd for $\mathrm{C}_{20} \mathrm{H}_{18} \mathrm{O}_{4} \mathrm{Na}[\mathrm{M}+\mathrm{Na}]^{+} 345.1103$, found 345.1107.

$7 \mathrm{~g}-d_{1}$<smiles>[2H]/C(=C\CC(COc1ccccc1)C(=O)Oc1ccccc1)OC(C)=O</smiles>

${ }^{1} \mathrm{H}$ NMR (400 MHz, $\left.\mathrm{CDCl}_{3}\right) \delta 7.94(\mathrm{~m}, 4 \mathrm{H}), 7.57(\mathrm{~m}, 2 \mathrm{H}), 7.45(\mathrm{~m}, 4 \mathrm{H}), 7.15(\mathrm{~d}, J=12.5 \mathrm{~Hz}, 0.25 \mathrm{H})$, $5.50(\mathrm{~m}, 1 \mathrm{H}), 5.23(\mathrm{t}, J=6.6 \mathrm{~Hz}, 1 \mathrm{H}), 2.80(\mathrm{~m}, 2 \mathrm{H}), 2.07(\mathrm{~s}, 3 \mathrm{H}) .{ }^{13} \mathrm{C}$ NMR $\left(100 \mathrm{MHz}, \mathrm{CDCl}_{3}\right) \delta 195.68$ (C), $168.09(\mathrm{C}), 137.56(\mathrm{CH}), 135.74(\mathrm{C}), 133.88(\mathrm{CH}), 128.87(\mathrm{CH}), 128.50(\mathrm{CH}), 111.35$ (t, CD), $111.36(\mathrm{CH}), 57.29(\mathrm{CH}), 27.40\left(\mathrm{CH}_{2}\right), 20.54\left(\mathrm{CH}_{3}\right)$.

$7 \mathrm{~g}-d_{2}$.

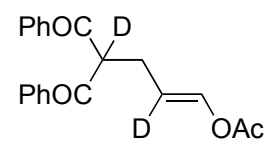

${ }^{1} \mathrm{H}$ NMR $\left(400 \mathrm{MHz}, \mathrm{CDCl}_{3}\right) \delta 7.94(\mathrm{~d}, J=7.8 \mathrm{~Hz}, 4 \mathrm{H}), 7.56(\mathrm{td}, J=7.4 \mathrm{~Hz}, 4 \mathrm{H}), 7.46-7.42(\mathrm{~m}, 4 \mathrm{H})$, 7.17-7.14 (m, 1H), 5.54-5.47 (m, 0.24H), $5.25(\mathrm{t}, J=6.6 \mathrm{~Hz}, 0.43 \mathrm{H}), 2.81-2.79(\mathrm{~m}, 2 \mathrm{H}), 2.02(\mathrm{~s}, 3 \mathrm{H}) ;{ }^{13} \mathrm{C}$ NMR (100 MHz, $\left.\mathrm{CDCl}_{3}\right) \delta 195.44$ (C), 195.41 (C), 168.08 (C), $137.40(\mathrm{CH}), 137.34(\mathrm{CH}), 135.96(\mathrm{C})$, $133.81(\mathrm{CH}), 129.10(\mathrm{CH}), 128.70(\mathrm{CH}), 111.51(\mathrm{CH}), 111.49(\mathrm{CH}), 111.24$ (t, $J=24.2 \mathrm{~Hz}, \mathrm{CD}), 57.42$ $(\mathrm{CH}), 57.05$ (t, $J=19.2 \mathrm{~Hz}, \mathrm{CD}), 27.54\left(\mathrm{CH}_{2}\right), 27.47\left(\mathrm{CH}_{2}\right), 20.74\left(\mathrm{CH}_{3}\right)$.

(Z)-4-(2,4,6-Trimethoxyphenyl)but-2-en-2-yl acetate $(7 \mathrm{~h})$.<smiles>COc1cc(OC)c(C/C=C(\C)OC(C)=O)c(OC)c1</smiles>

Compound 7h was synthesized following the general procedure (Scheme 3) starting from $\mathbf{1 h}$ (51 mg, 0.45 $\mathrm{mmol})$ and 16 (113 mg, $0.67 \mathrm{mmol})$. The residue was purified by chromatography ( $8: 1$ hexane-EtOAc) to give $7 \mathbf{h}(26 \mathrm{mg}, 21 \%)$ as a pale yellow oil. ${ }^{1} \mathrm{H}$ NMR $\left(400 \mathrm{MHz}, \mathrm{CDCl}_{3}\right) \delta 6.13(\mathrm{~s}, 2 \mathrm{H}), 5.74(\mathrm{t}, J=6.4$ $\mathrm{Hz}, 1 \mathrm{H}), 4.29(\mathrm{~d}, J=6.4 \mathrm{~Hz}, 2 \mathrm{H}), 3.82(\mathrm{~s}, 6 \mathrm{H}), 2.15$ (s, 3H), $1.99(\mathrm{~s}, 3 \mathrm{H}) ;{ }^{13} \mathrm{C} \mathrm{NMR}\left(100 \mathrm{MHz}, \mathrm{CDCl}_{3}\right) \delta$ $167.6(\mathrm{C}), 158.6(\mathrm{C}), 144.2(\mathrm{C}), 122.6(\mathrm{CH}), 109.0(\mathrm{C}), 92.2(\mathrm{CH}), 90.7(\mathrm{C}), 55.3\left(\mathrm{CH}_{3}\right), 20.8\left(\mathrm{CH}_{3}\right), 19.4$ $\left(\mathrm{CH}_{3}\right), 18.9\left(\mathrm{CH}_{2}\right)$. HRMS-ESI $m / z$ calcd for $\mathrm{C}_{30} \mathrm{H}_{40} \mathrm{O}_{10} \mathrm{Na}[\mathrm{M}+\mathrm{Na}]^{+} 560.2600$, found 560.2600.

1,3-Diphenyl-2-(1-phenylprop-2-ynyl)propane-1,3-dione (13).<smiles>C#CC(c1ccccc1)C(C(=O)Oc1ccccc1)C(=O)c1ccccc1</smiles> 
Compound 13 was synthesized following the general procedure (Table 1, entry 7) starting from 1a (30 $\mathrm{mg}, 0.10 \mathrm{mmol})$ and $12 \mathrm{a}(35 \mathrm{mg}, 0.16 \mathrm{mmol})$. The residue was purified by chromatography $(8: 1$ hexaneEtOAc) to give 5a (35 mg, 99\%) as a white solid. The spectroscopic data are consistent with those described. $^{8}$

\section{2-Benzoyl-1,3-diphenylpentane-1,4-dione (17).}<smiles>CC(=O)C(c1ccccc1)C(C(=O)c1ccccc1)C(=O)c1ccccc1</smiles>

Compound 17 was synthesized starting from $1 \mathrm{a}(30 \mathrm{mg}, 0.10 \mathrm{mmol})$ and $12 \mathrm{a}(35 \mathrm{mg}, 0.16 \mathrm{mmol})$ in $\mathrm{CH}_{2} \mathrm{Cl}_{2}(1.5 \mathrm{~mL})$ with $\mathrm{AgSbF}_{6}(5 \mathrm{~mol} \%)$. The reaction mixture was stirred at room temperature for $32 \mathrm{~h}$. The mixture was filtered trough silica gel with $\mathrm{CH}_{2} \mathrm{Cl}_{2}$ and the solvents evaporated. The residue was chromatographed (8:1 hexane-EtOAc) to give compound 20 (36 mg, 97\%) as a pale yellow solid: $\mathrm{mp}$ 160-162 ${ }^{\circ} \mathrm{C} .{ }^{1} \mathrm{H}$ NMR (400 MHz, $\left.\mathrm{CDCl}_{3}\right) \delta 7.91(\mathrm{~m}, 2 \mathrm{H}), 7.64(\mathrm{~m}, 1 \mathrm{H}), 7.47(\mathrm{~m}, 1 \mathrm{H}), 7.41(\mathrm{~m}, 1 \mathrm{H}), 7.33$ (m, 2H), $7.25(\mathrm{~m}, 2 \mathrm{H}), 7.21-7.07(\mathrm{~m}, 5 \mathrm{H}), 6.13(\mathrm{~d}, \mathrm{~J}=10.9 \mathrm{~Hz}, 1 \mathrm{H}), 4.94(\mathrm{~d}, \mathrm{~J}=10.9 \mathrm{~Hz}, 1 \mathrm{H}), 2.22$ (s, $3 \mathrm{H}) ;{ }^{13} \mathrm{C}$ NMR (100 MHz, $\mathrm{CDCl}_{3}$, Pendant) $\delta 206.46$ (C), $195.44(\mathrm{C}), 194.45$ (C), $136.59(\mathrm{C}), 136.25$ (C), $134.04(\mathrm{C}), 133.33(\mathrm{CH}), 133.26(\mathrm{CH}), 129.10(\mathrm{CH}), 128.96(\mathrm{CH}), 128.62(\mathrm{CH}), 128.59(\mathrm{CH}), 128.57$ $(\mathrm{CH}), 128.37(\mathrm{CH}), 127.97(\mathrm{CH}), 59.95(\mathrm{CH}), 59.85(\mathrm{CH}), 29.12(\mathrm{CH})$; HRMS-ESI $\mathrm{m} / z$ calcd for $\mathrm{C}_{24} \mathrm{H}_{20} \mathrm{O}_{3} \mathrm{Na}[\mathrm{M}+\mathrm{Na}]^{+} 356.1421$, found 356.14 .

(1) Eberle, M. K.; Kahle, G. G. J. Am. Chem. Soc. 1977, 99, 6038-6042.

(2) Rodriguez, D.; Pérez Sestelo, J.; Sarandeses, L. A. J. Org. Chem. 2003, 68, 2518-2520.

(3) Zimmerman, H. E.; Baker, M. R.; Bottner, R. C.; Morrissey, M. M.; Murphy, S. J. Am. Chem. Soc. 1993, $115,459-466$.

(4) Chakraborti, A. S.; Sharma, L.; Gulhane, R.; Shivani Tetrahedron 2003, 49, 7661-7668.

(5) Trahanovsky, W. S.; Emeis, S. J. Am. Chem. Soc. 1974, 96, 3773-3777.

(6) Trost, B. M.; Hiroi, K.; Jungheim, L. J. Org. Chem. 1980, 45, 1839-1847.

(7) Farneth, W. E.; Brauman, J. I. J. Am. Chem. Soc. 1976, 98, 7891-7898.

(8) Sanz, R.; Miguel, D.; Martínez, A.; Álvarez-Gutiérrez, J. M.; Rodríguez, F. Org. Lett. 2007, 9, 727-730. 
PhOC $\underbrace{\mathrm{OAC}}_{\mathbf{5 a}} \mathrm{Ph}$

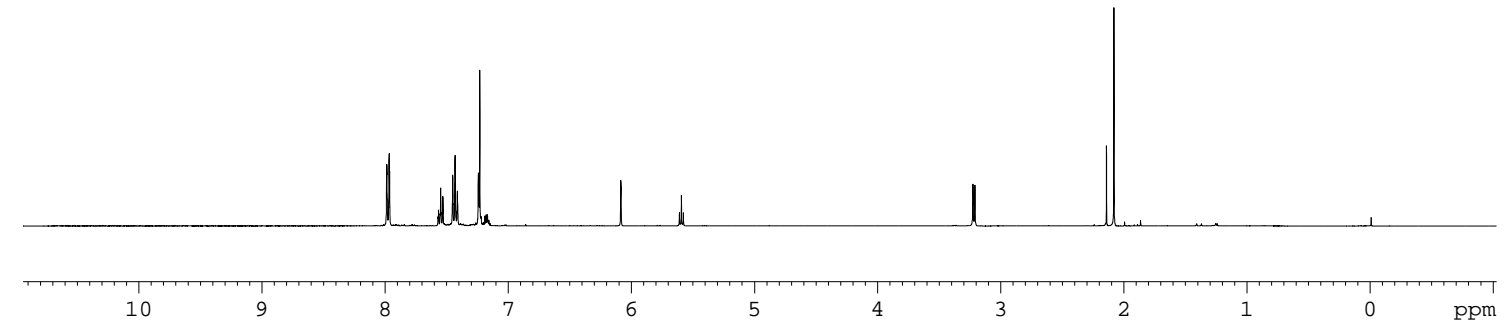

\footnotetext{
PhOC
}

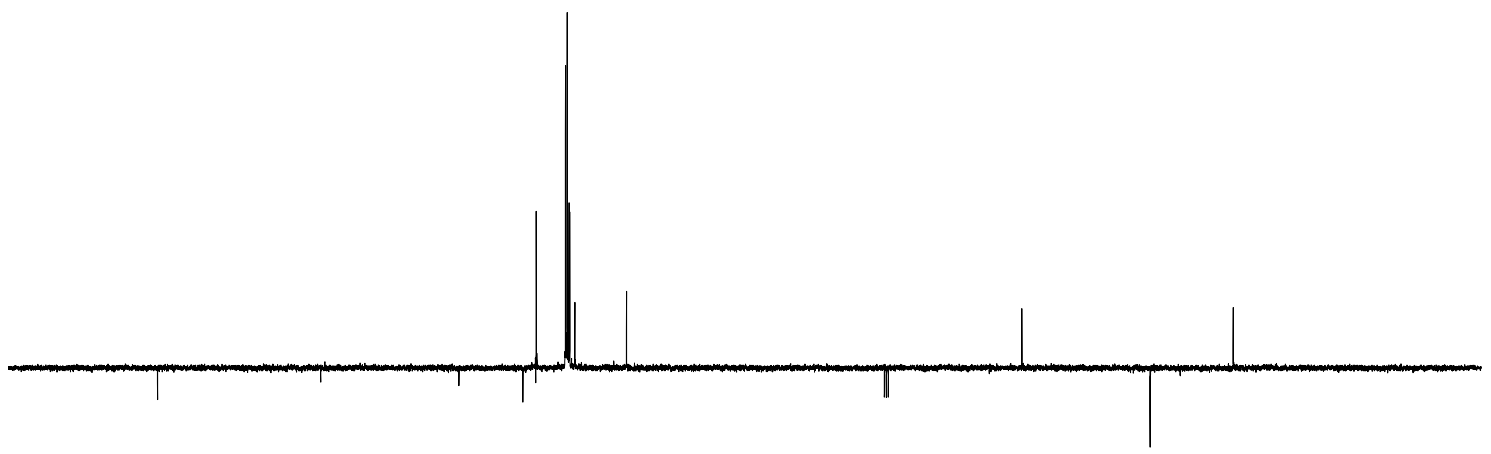

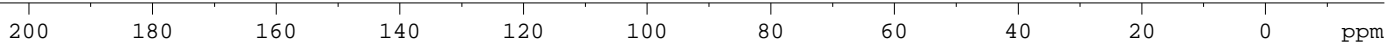



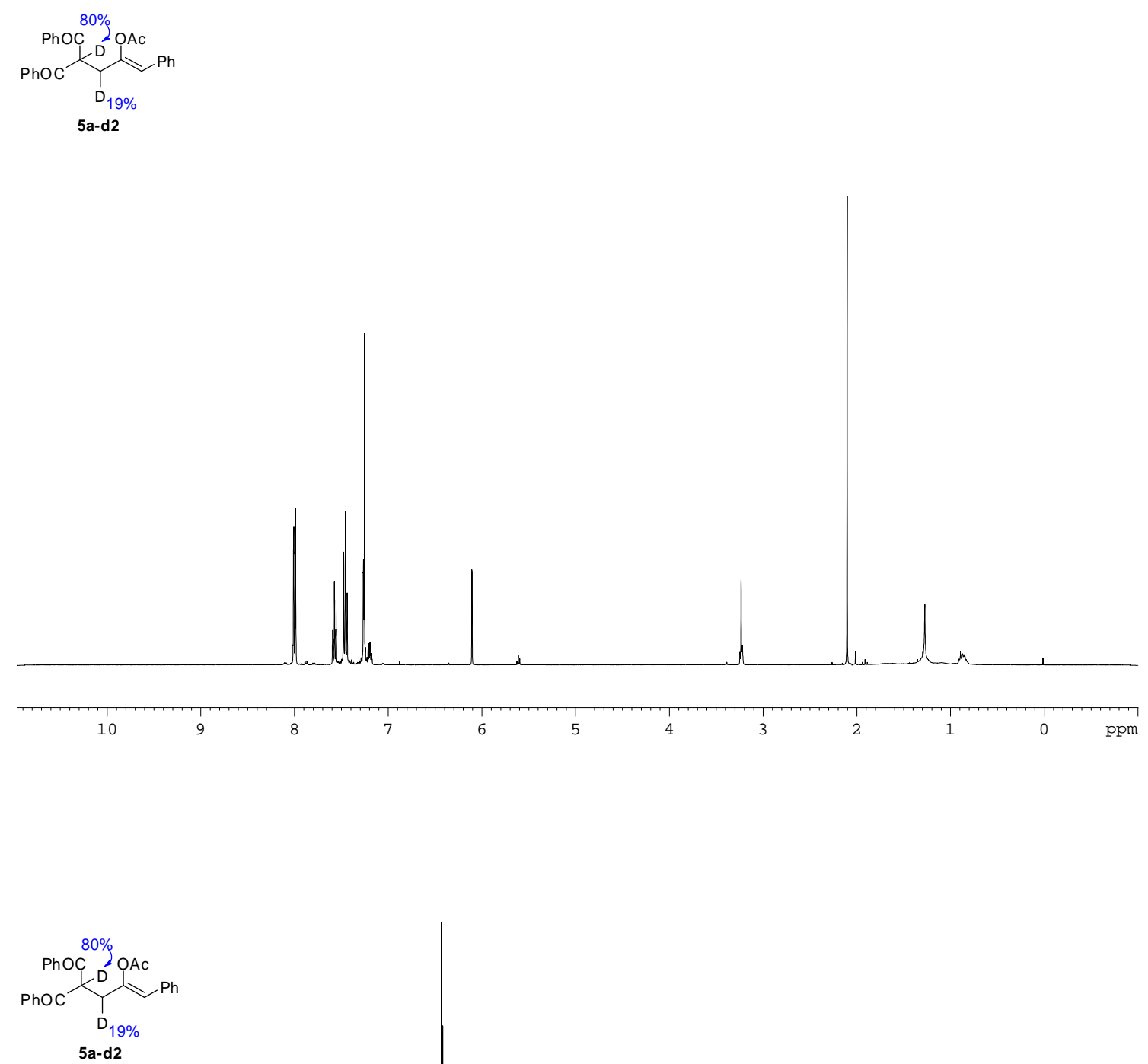
MeOC$\underbrace{\mathrm{OAC}}_{\mathbf{5 b}}$

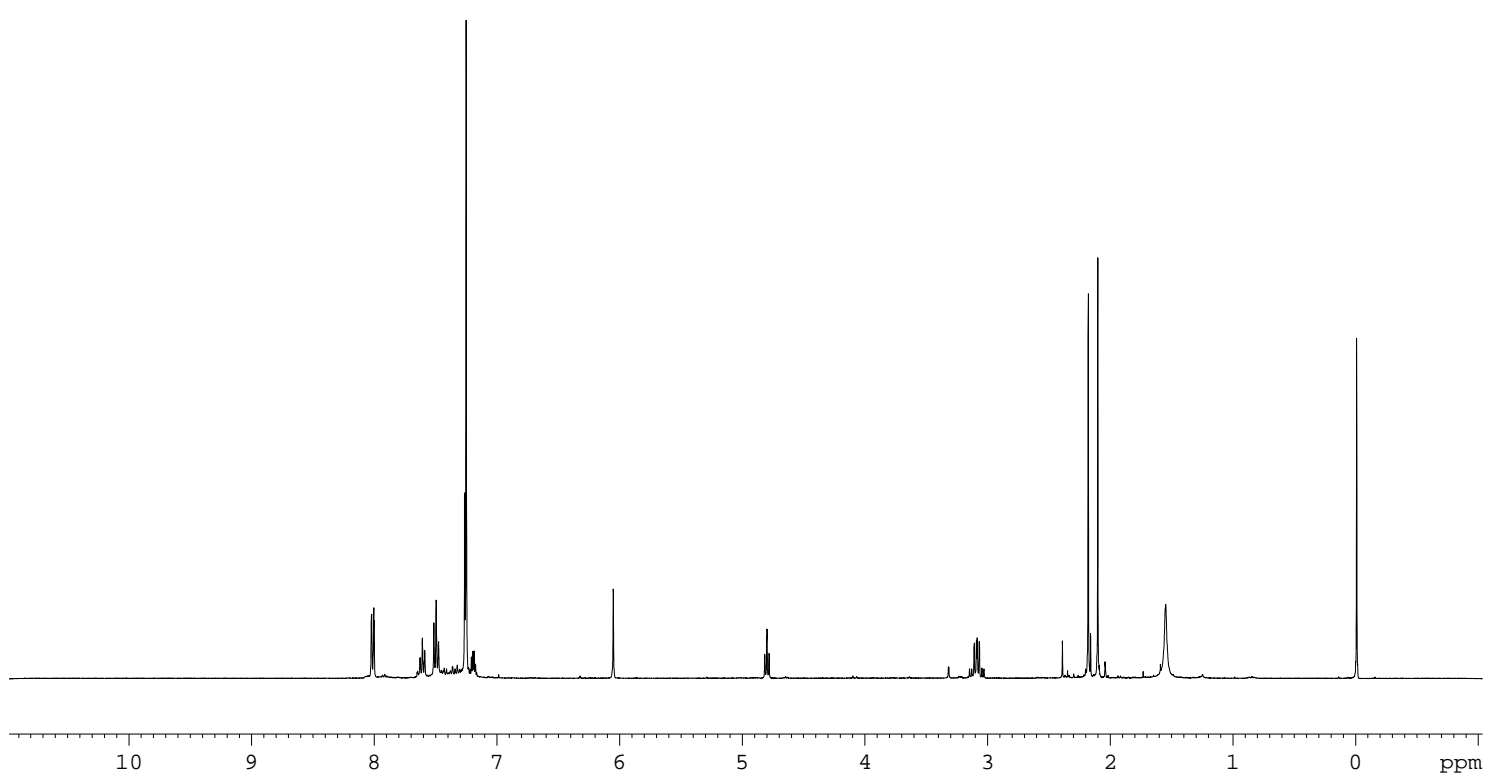

PhOC $\underbrace{\mathrm{MAC}}_{\mathbf{5 b}} \mathrm{Ph}$

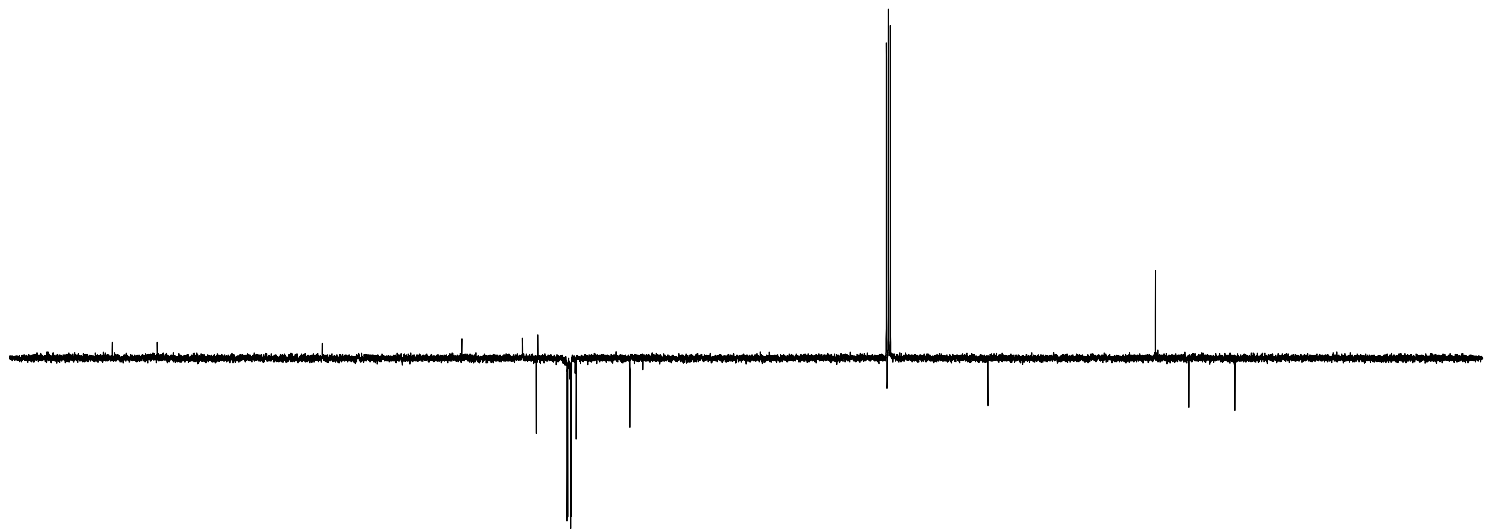

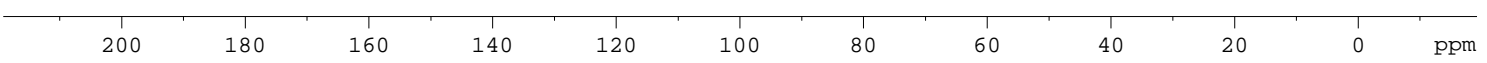


PhOc $\underbrace{\mathrm{OBz}}_{\mathbf{5 c}}$

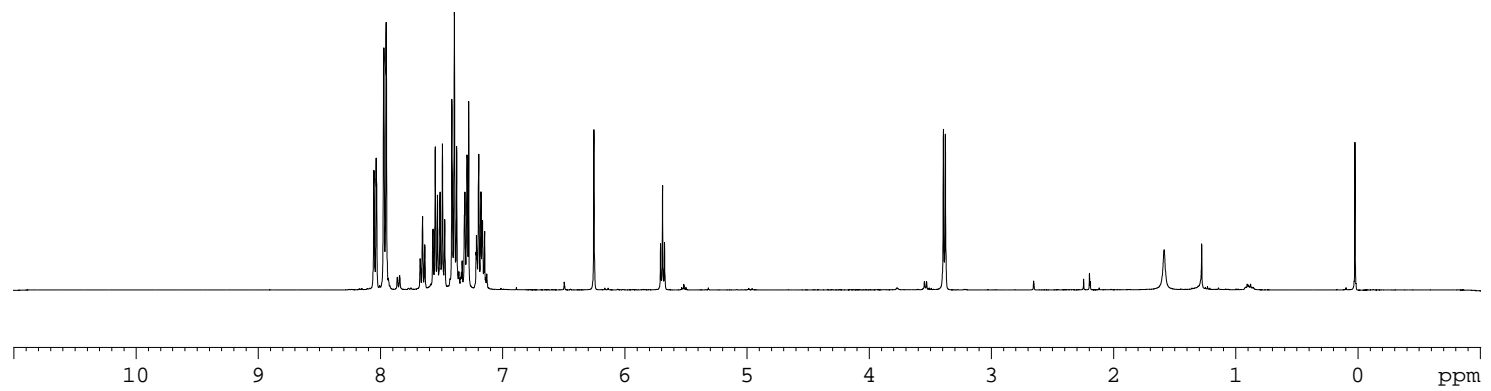

PhOC $\underbrace{\mathrm{OBz}}_{\mathbf{5 c}} \mathrm{Ph}$

200

180

160

140

120

100

80

60

40

20

$\odot \quad$ ppm 


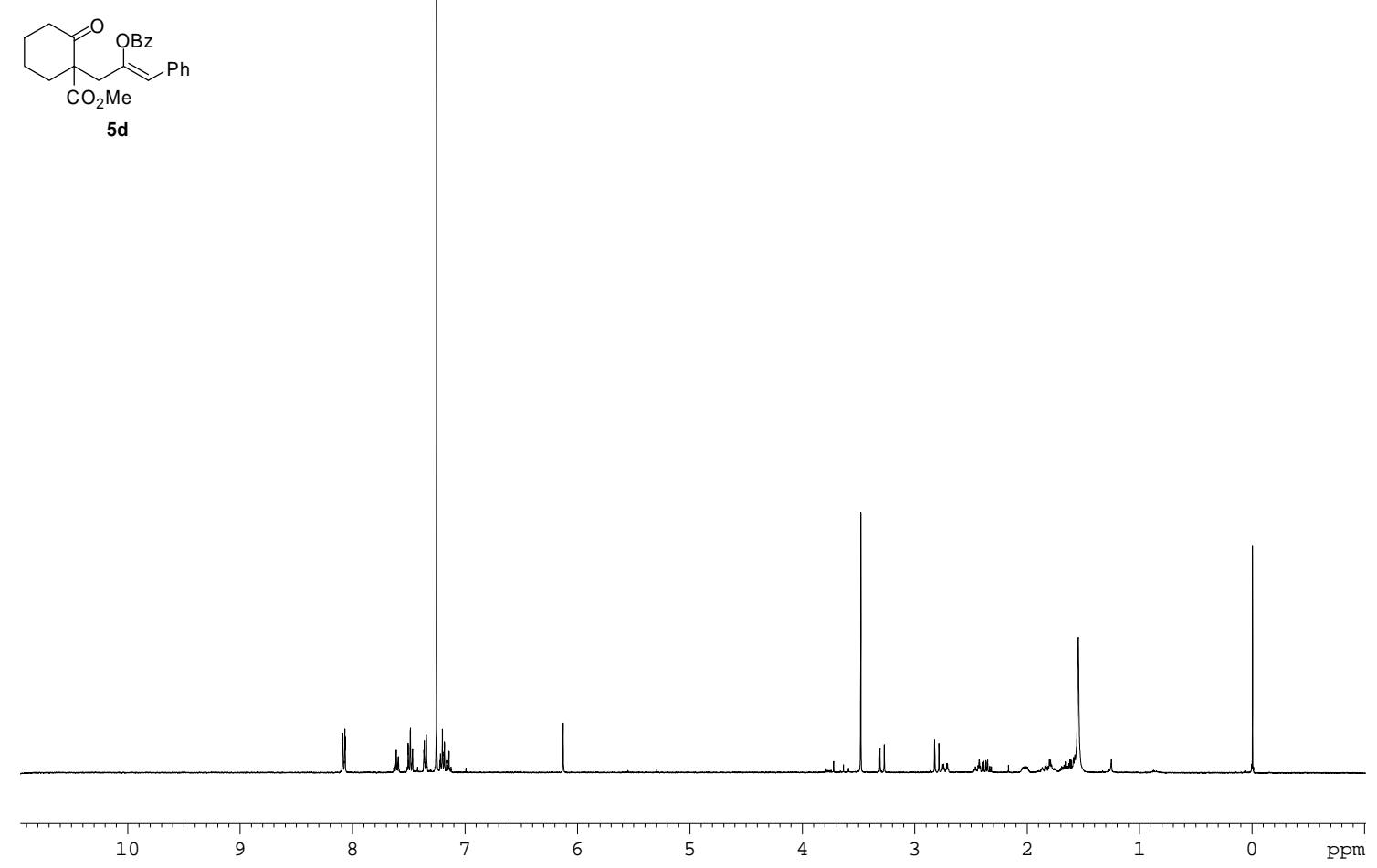

$\overbrace{\mathrm{CO}_{2} \mathrm{Me}}^{\mathrm{O}} \mathrm{OBz}$

$5 d$
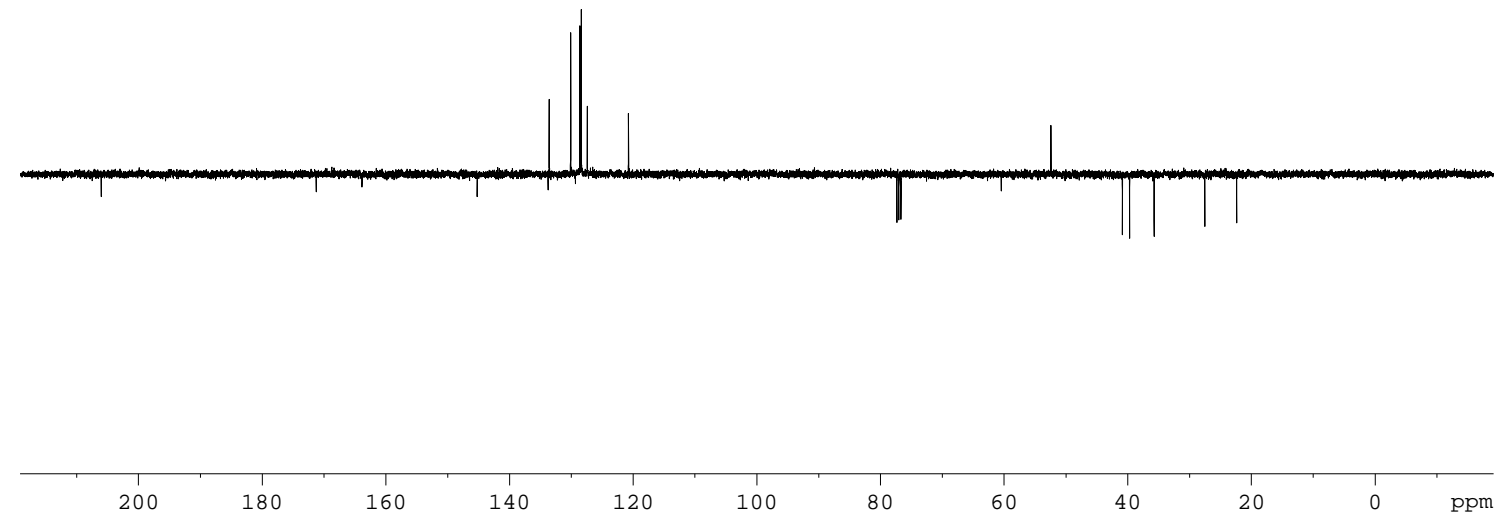


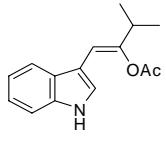

5

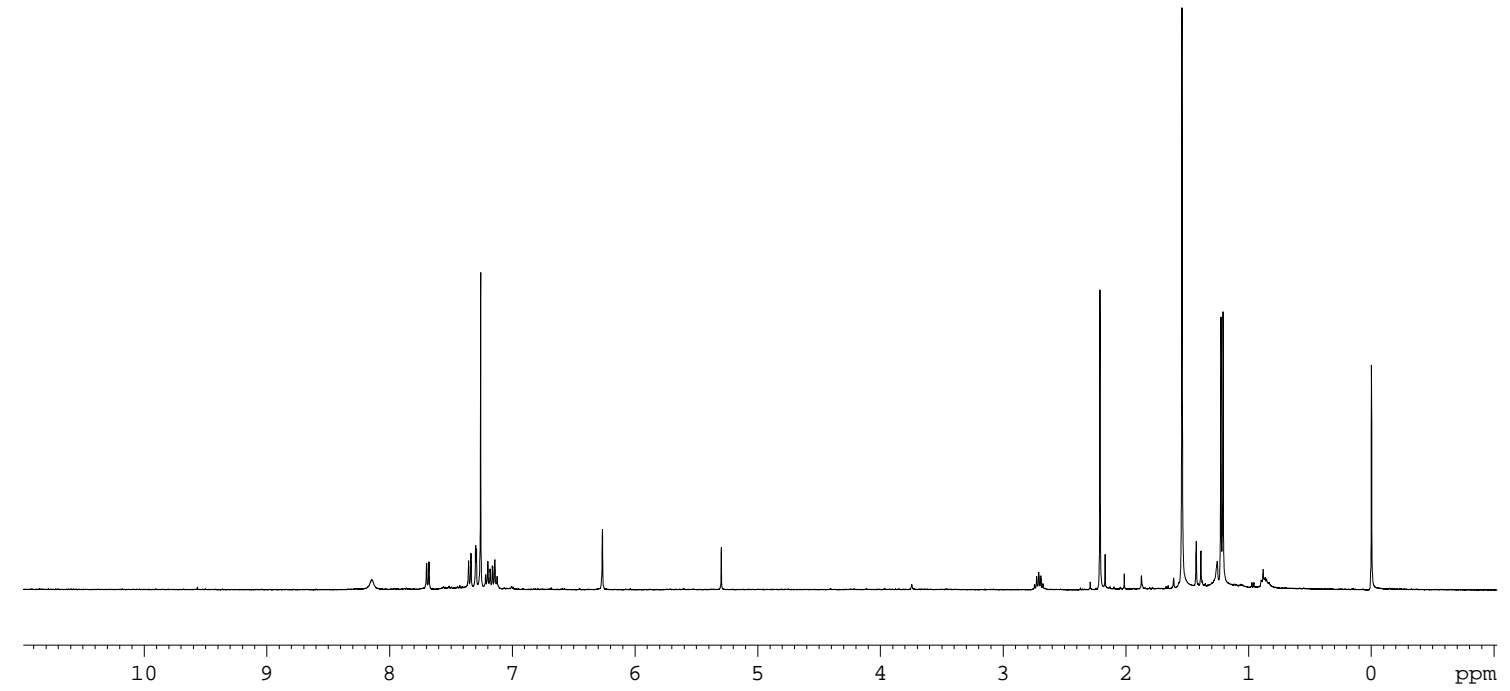

$\underbrace{}_{5 e}$

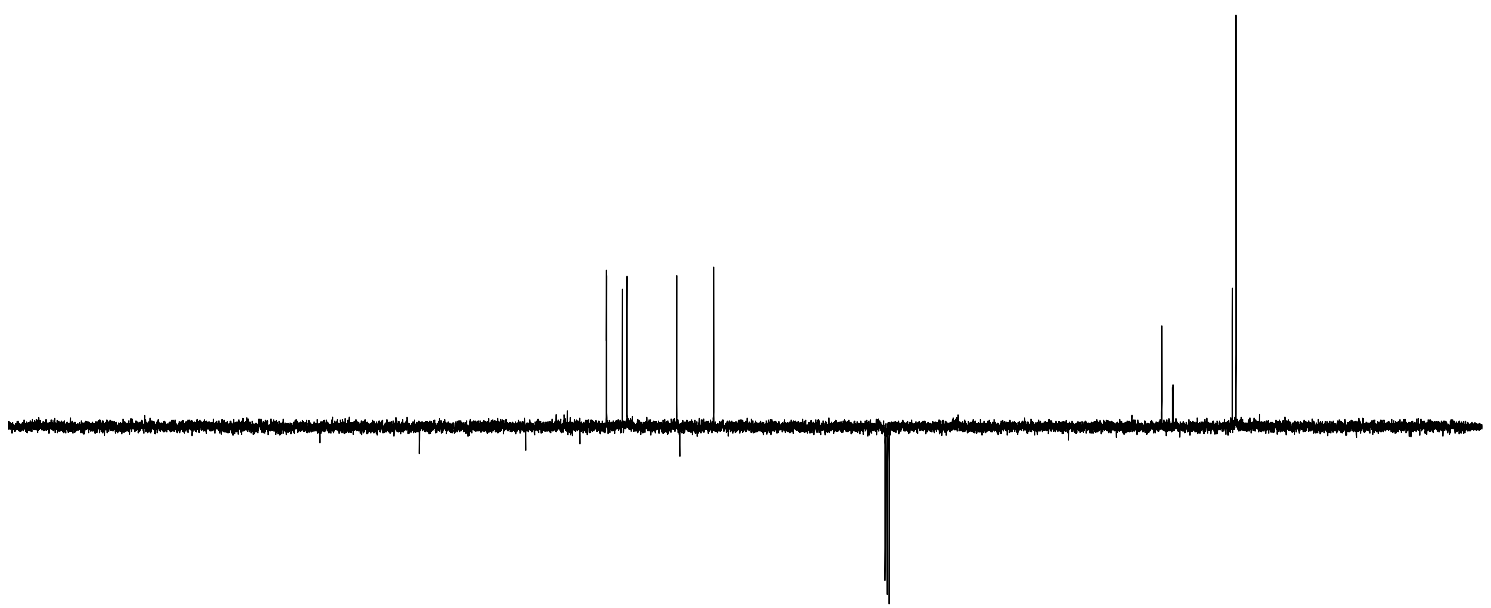


Phoc Opiv

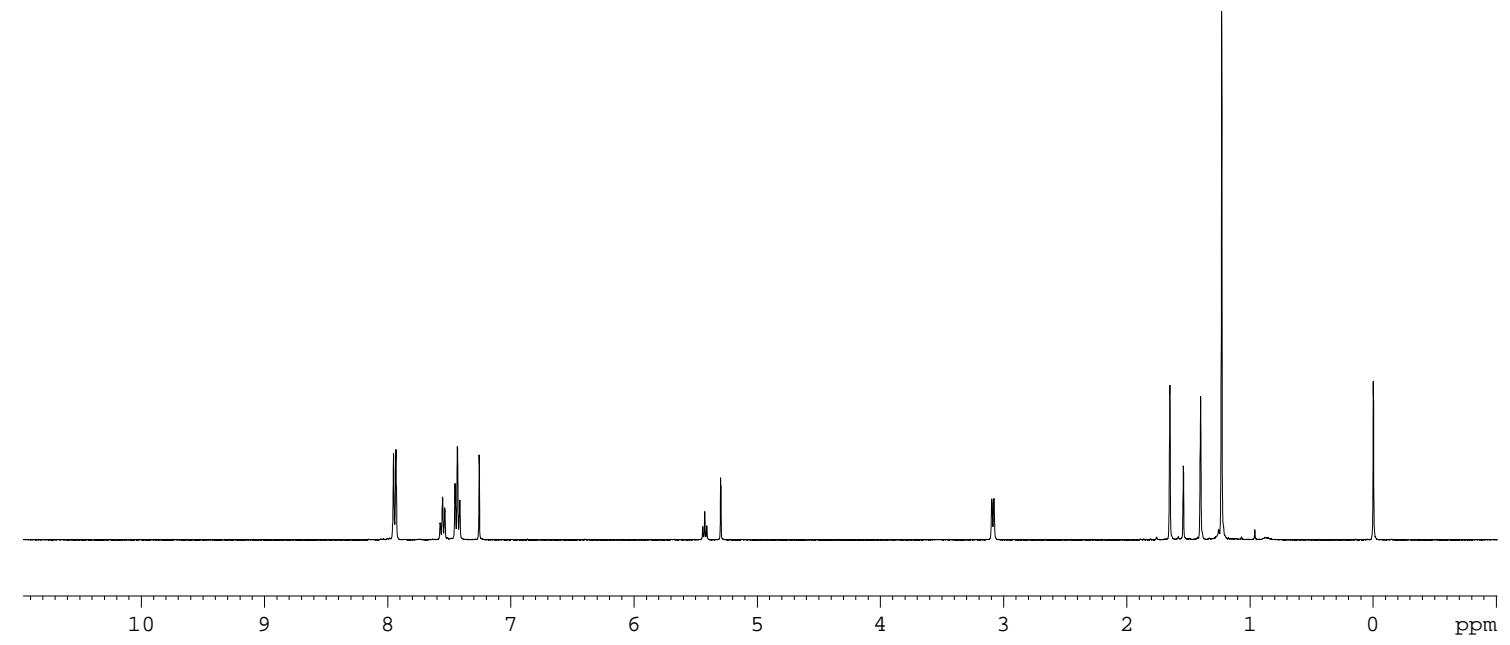

Phoc Opiv

$5 f$

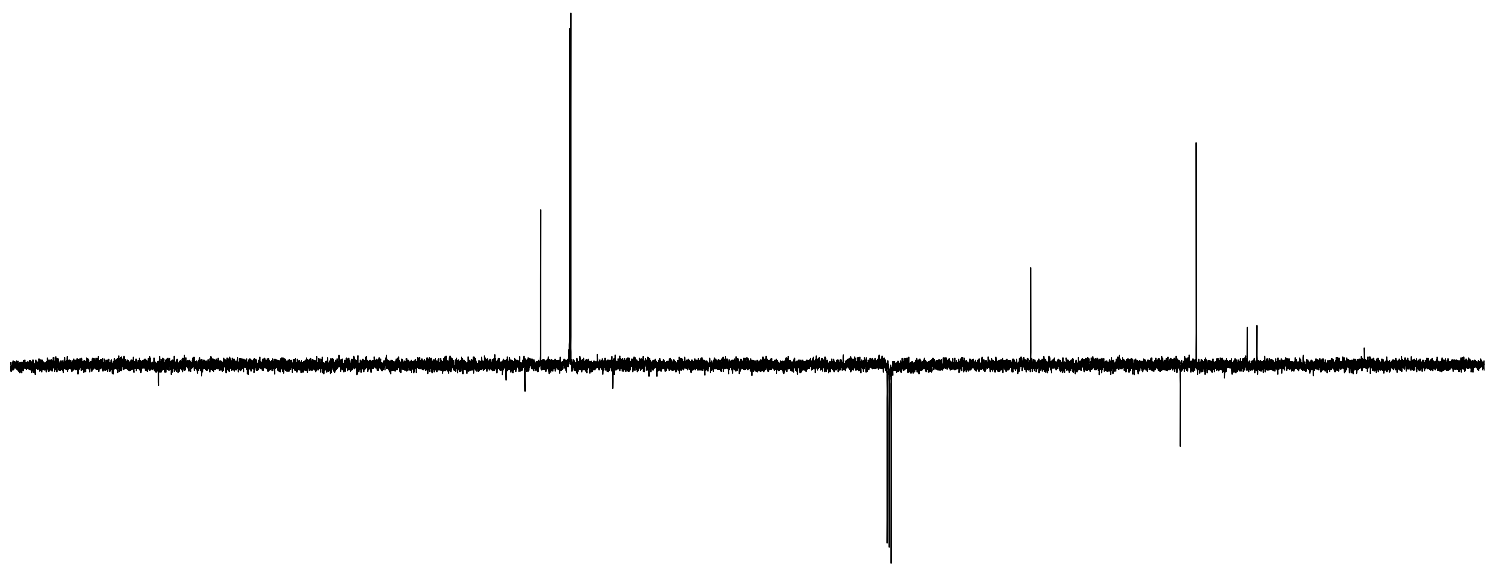

200

160

140

120

100

80

60

40

20

ppm 
5g

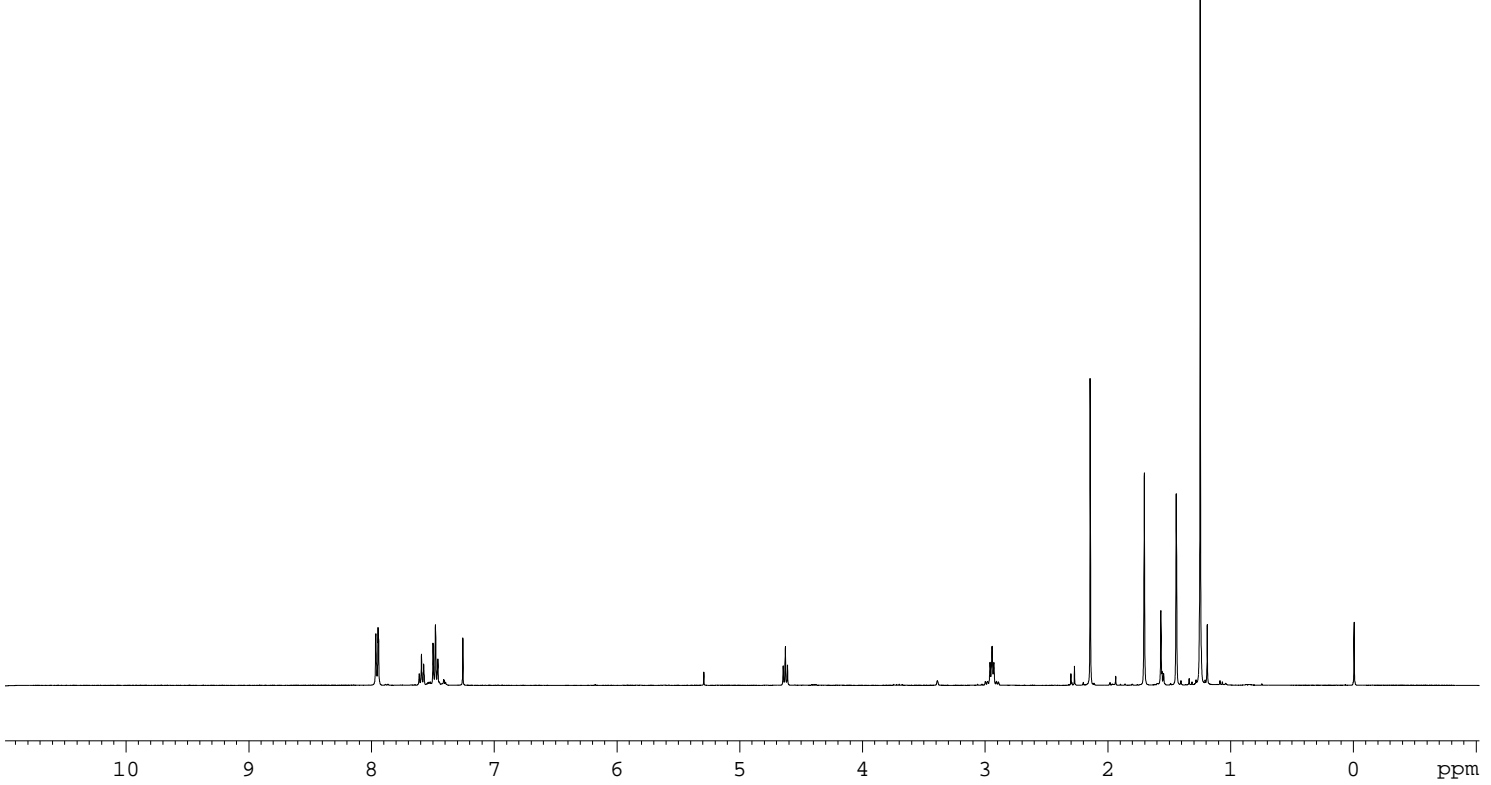

MeOc

$5 \mathrm{~g}$

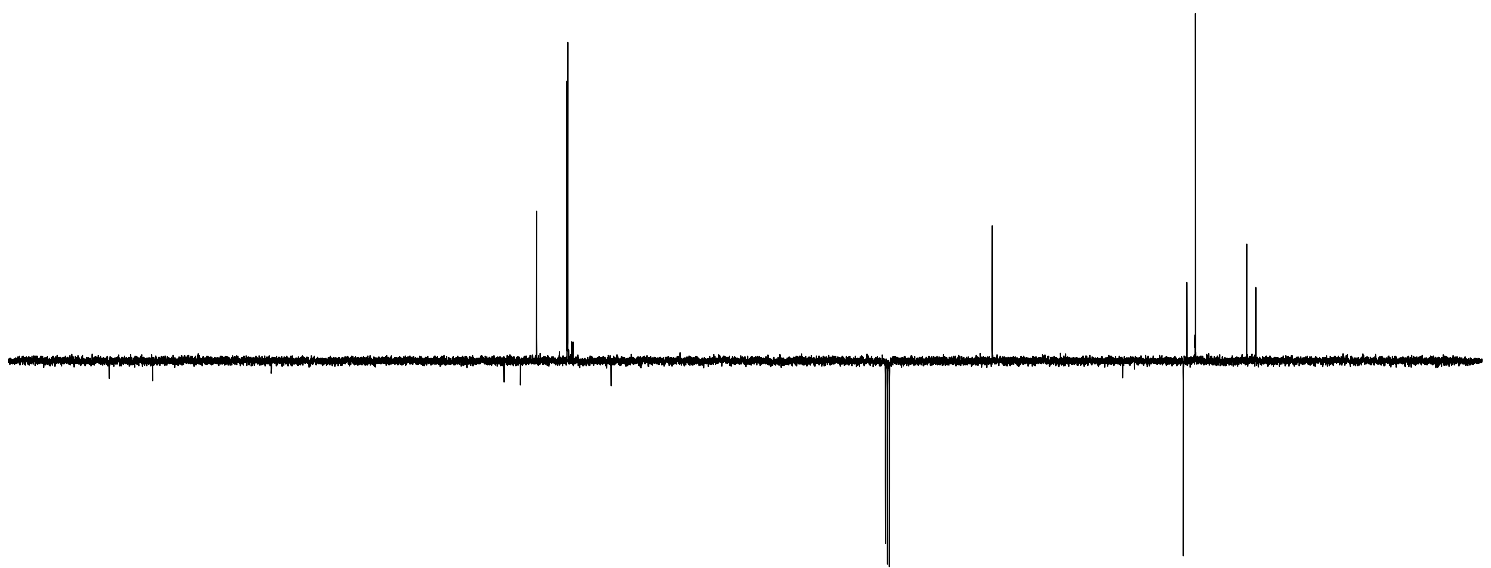

200

180

$16 \odot$

140

120

100

80

60

40

20

$\odot \quad$ ppm 
(nht)
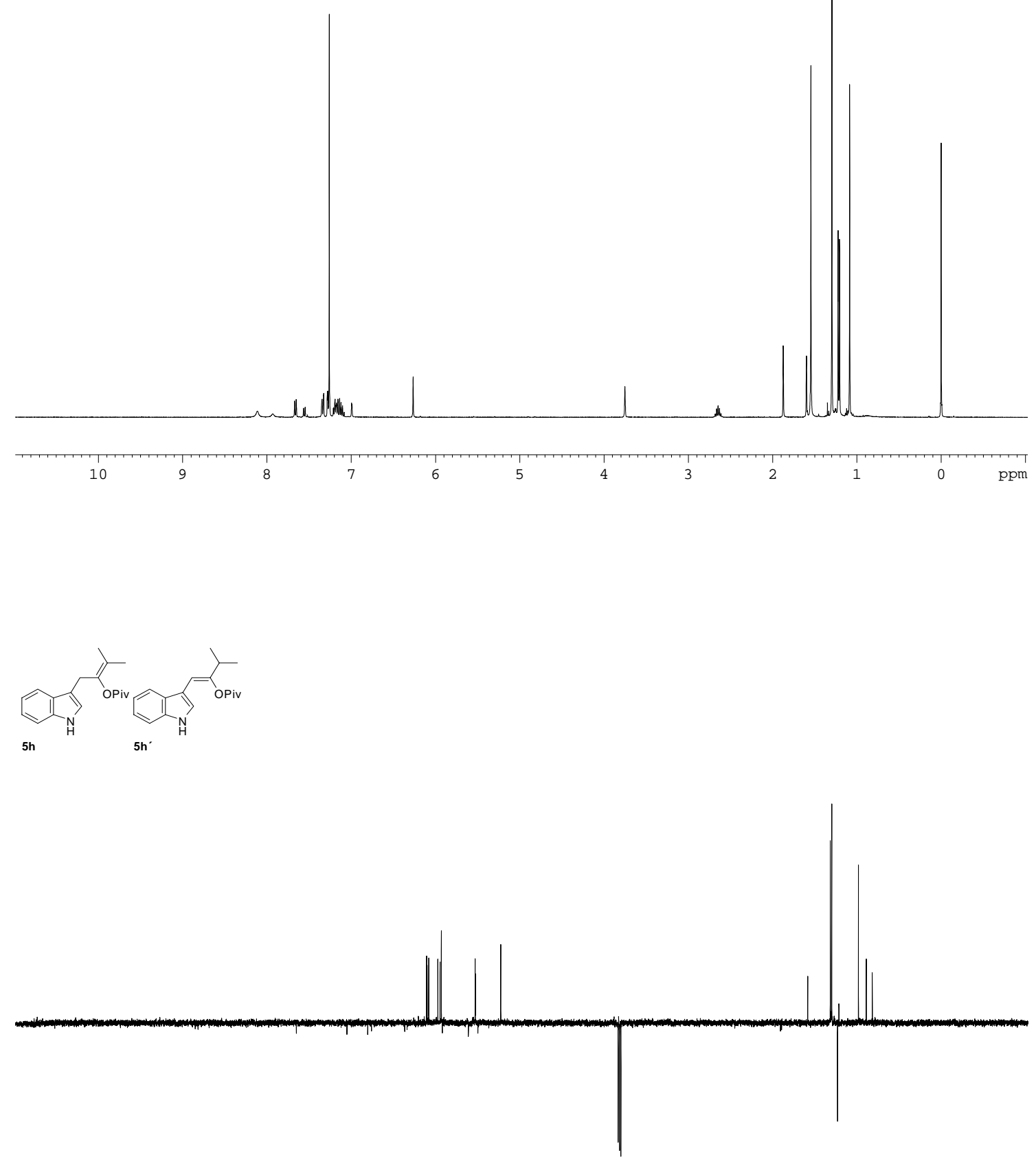

200

180 

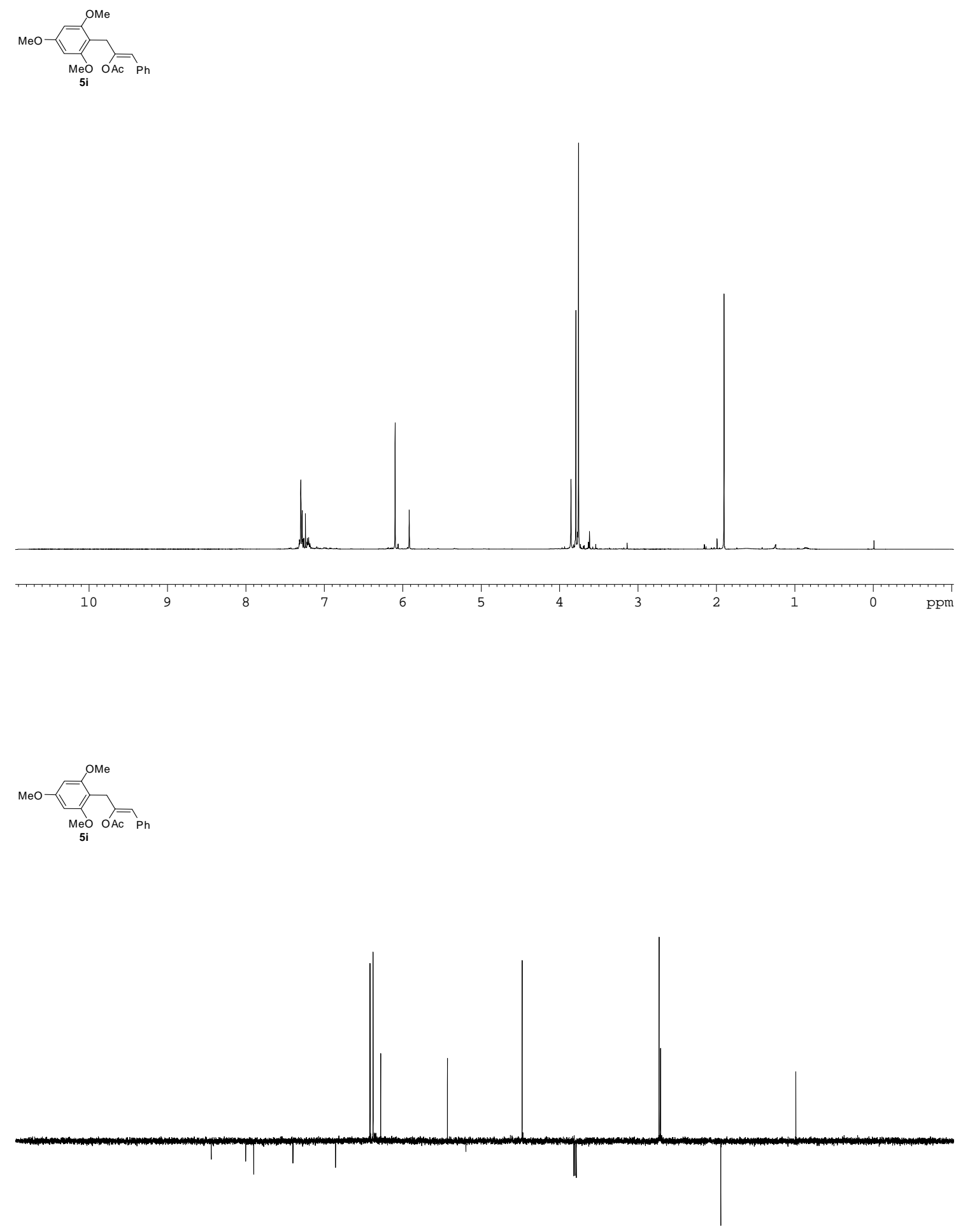

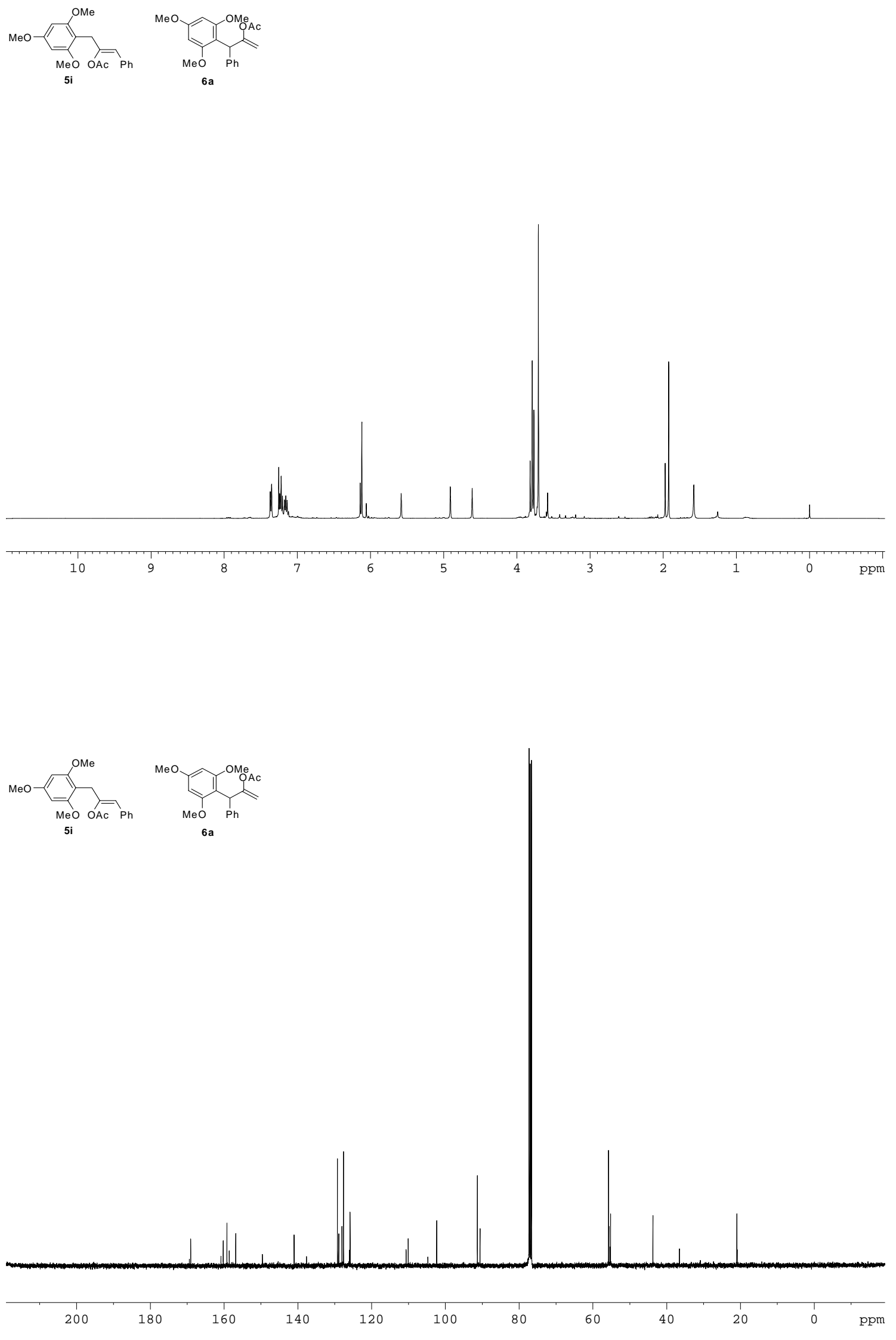

S-22 


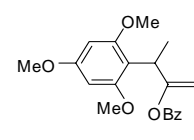

6b

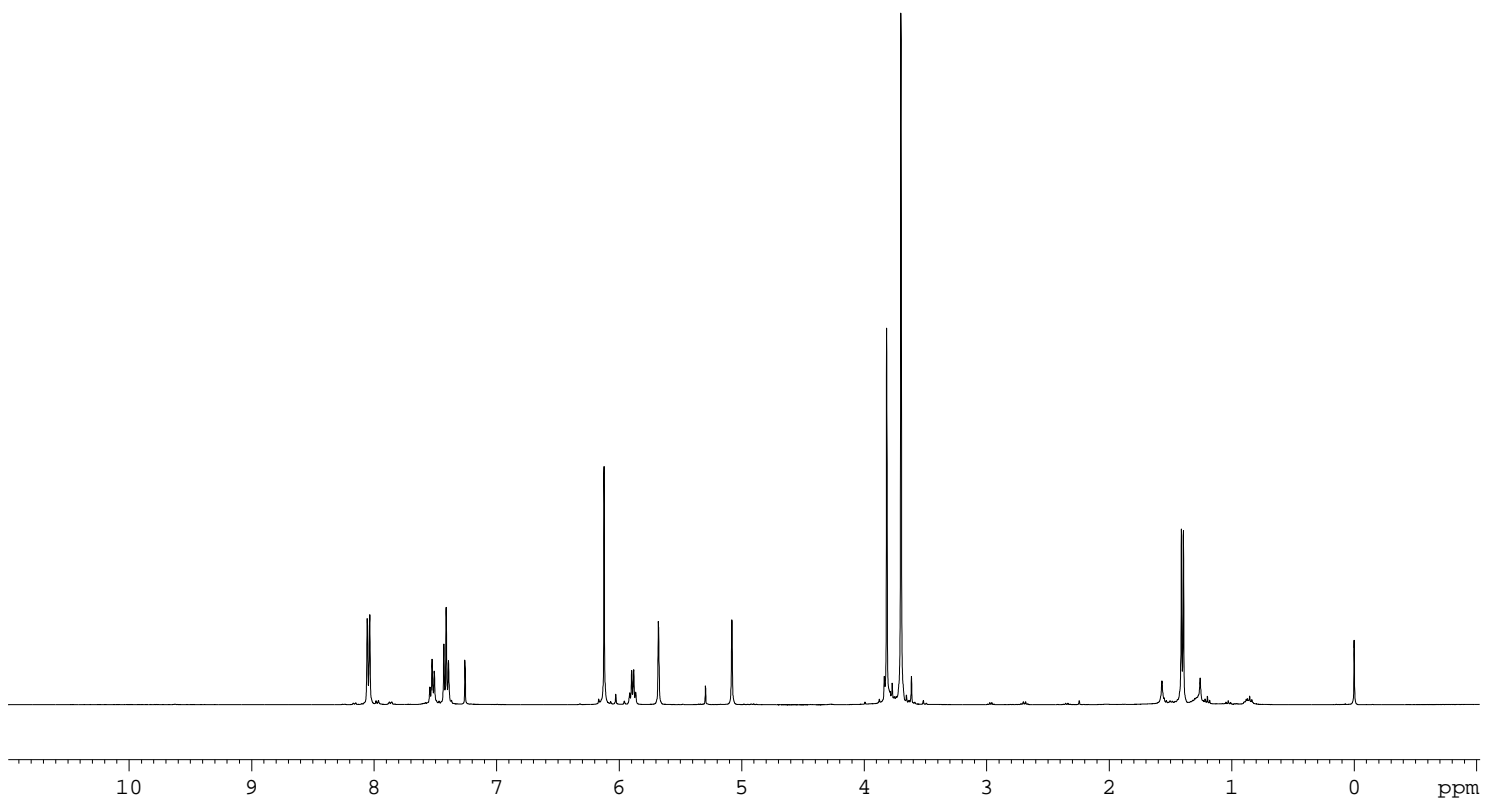

MeO

6b

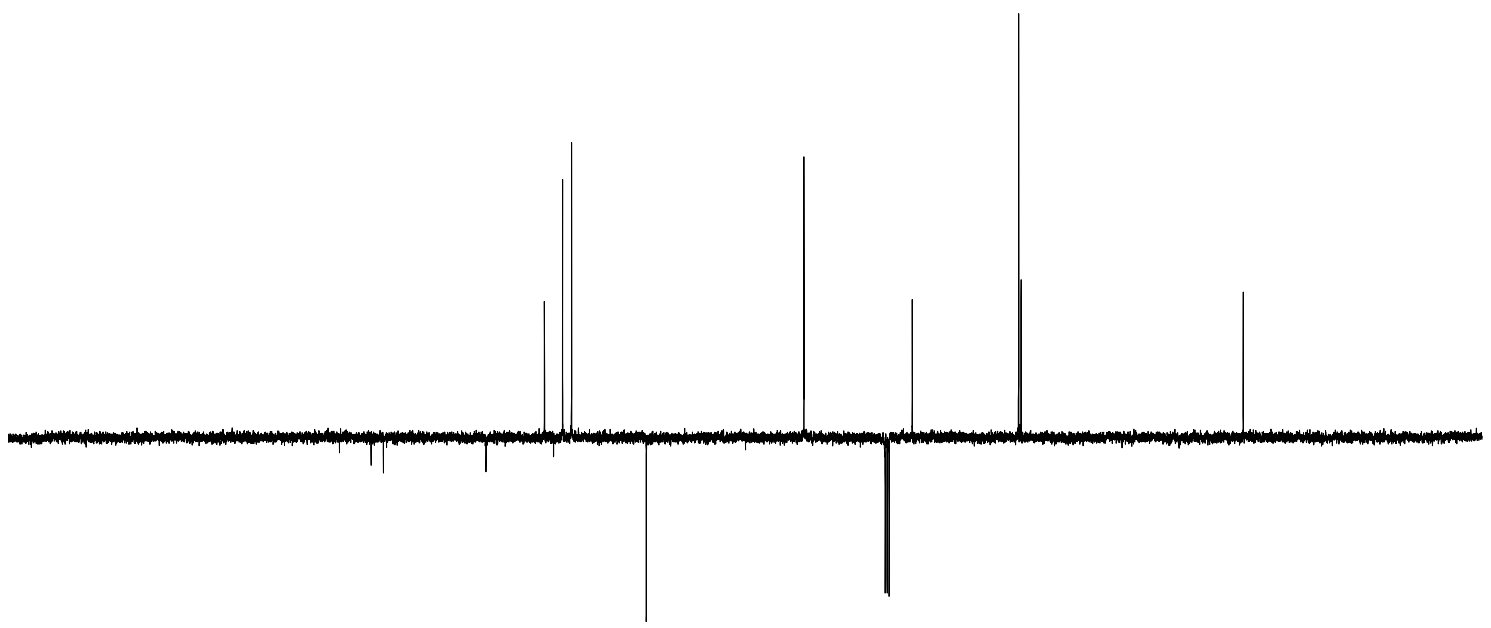

200

180

160

140

120

100

80

60

40

20

$\odot \quad$ ppm 
$\mathrm{O}_{\mathrm{OBZ}}$

$6 c$

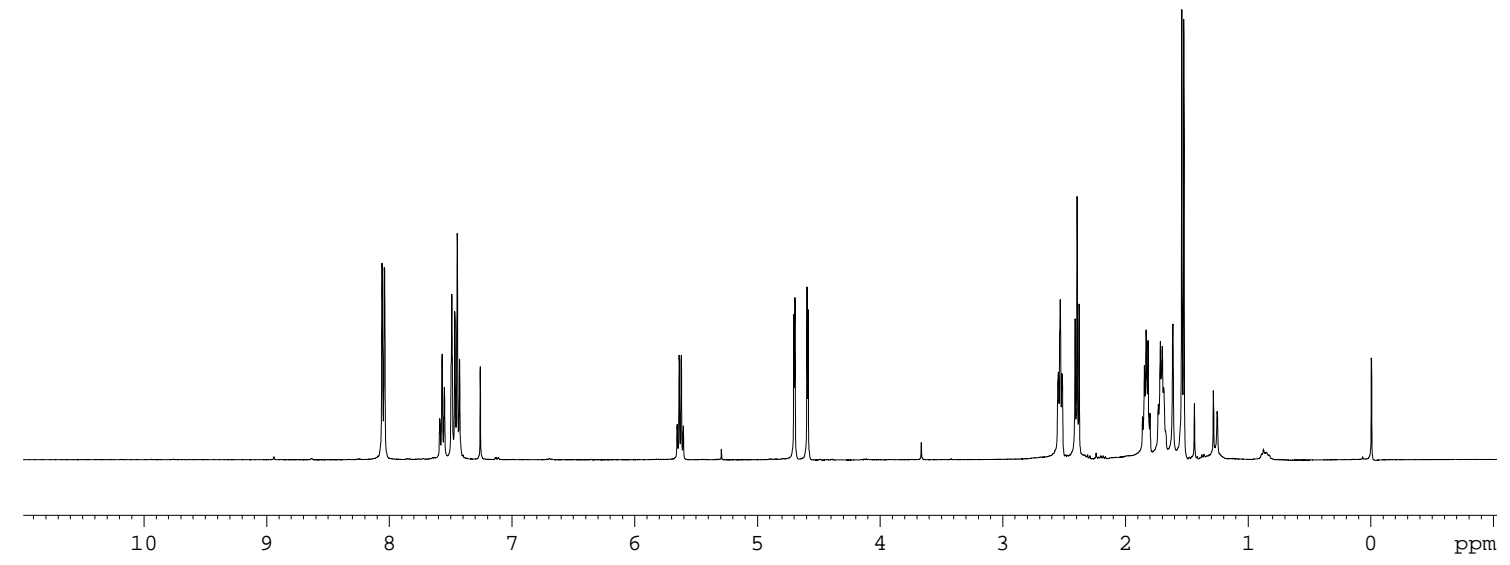

$\mathrm{O}_{\mathrm{OBz}}$

$6 c$

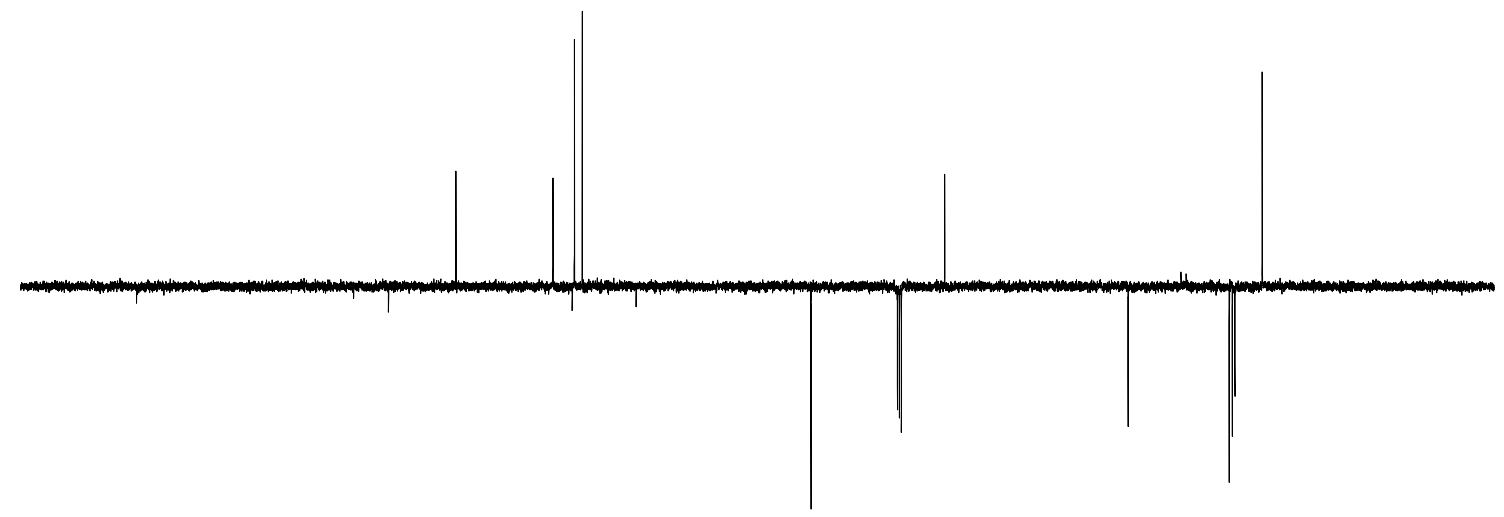

200

180

160

$140 \quad 120$

100

80

60

40

20

ppm 
$\mathrm{MeO}_{2} \mathrm{C}$

$7 a$

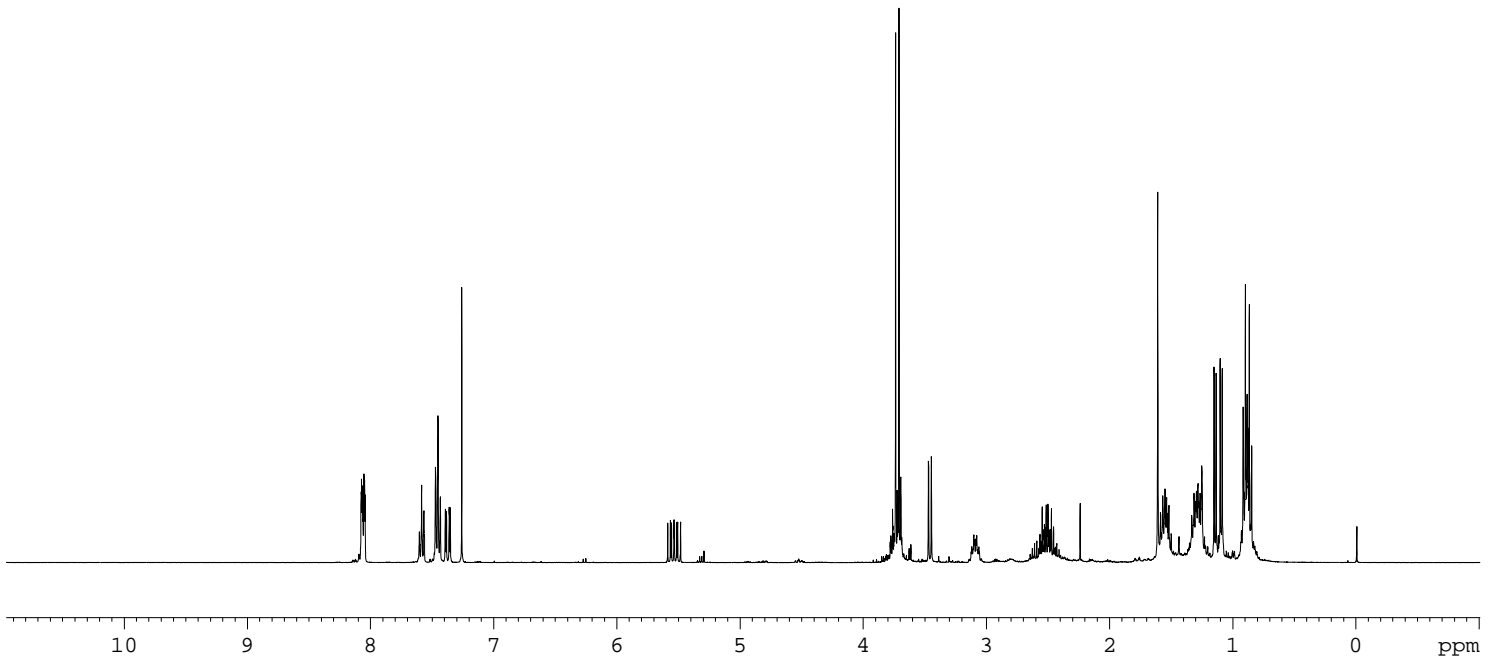

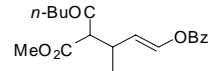

$7 a$

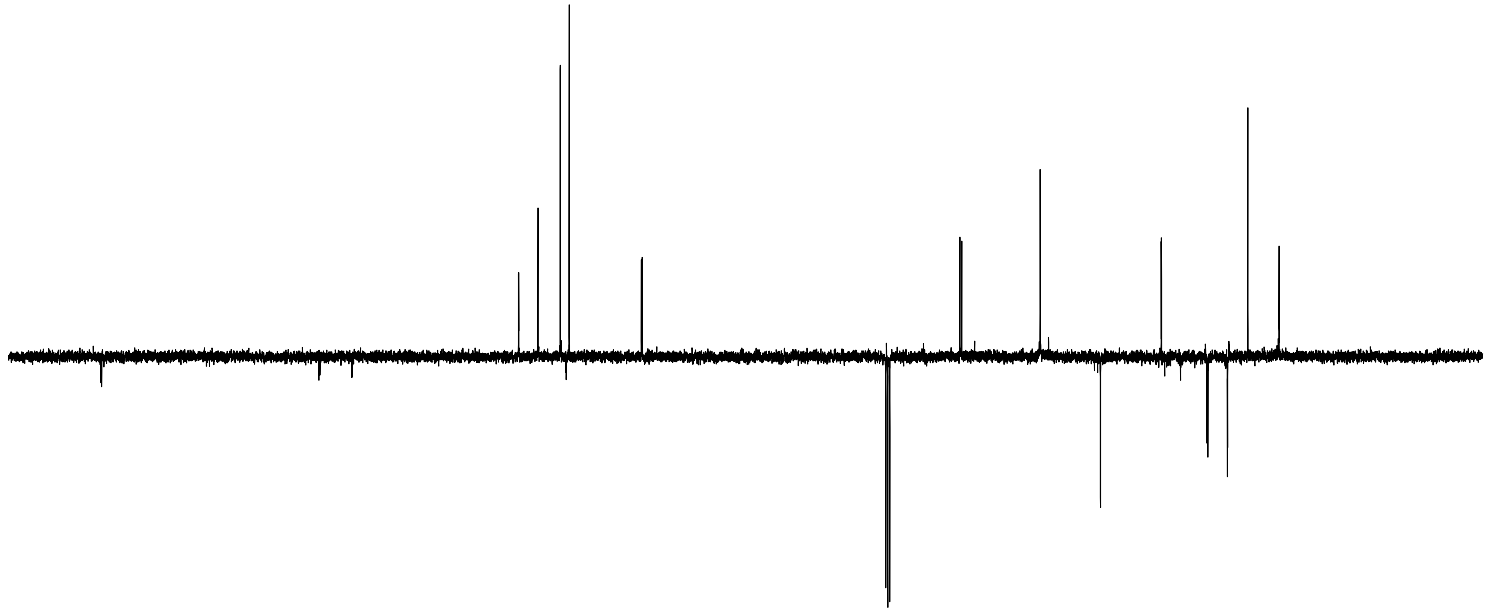

200 


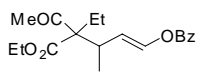

7b

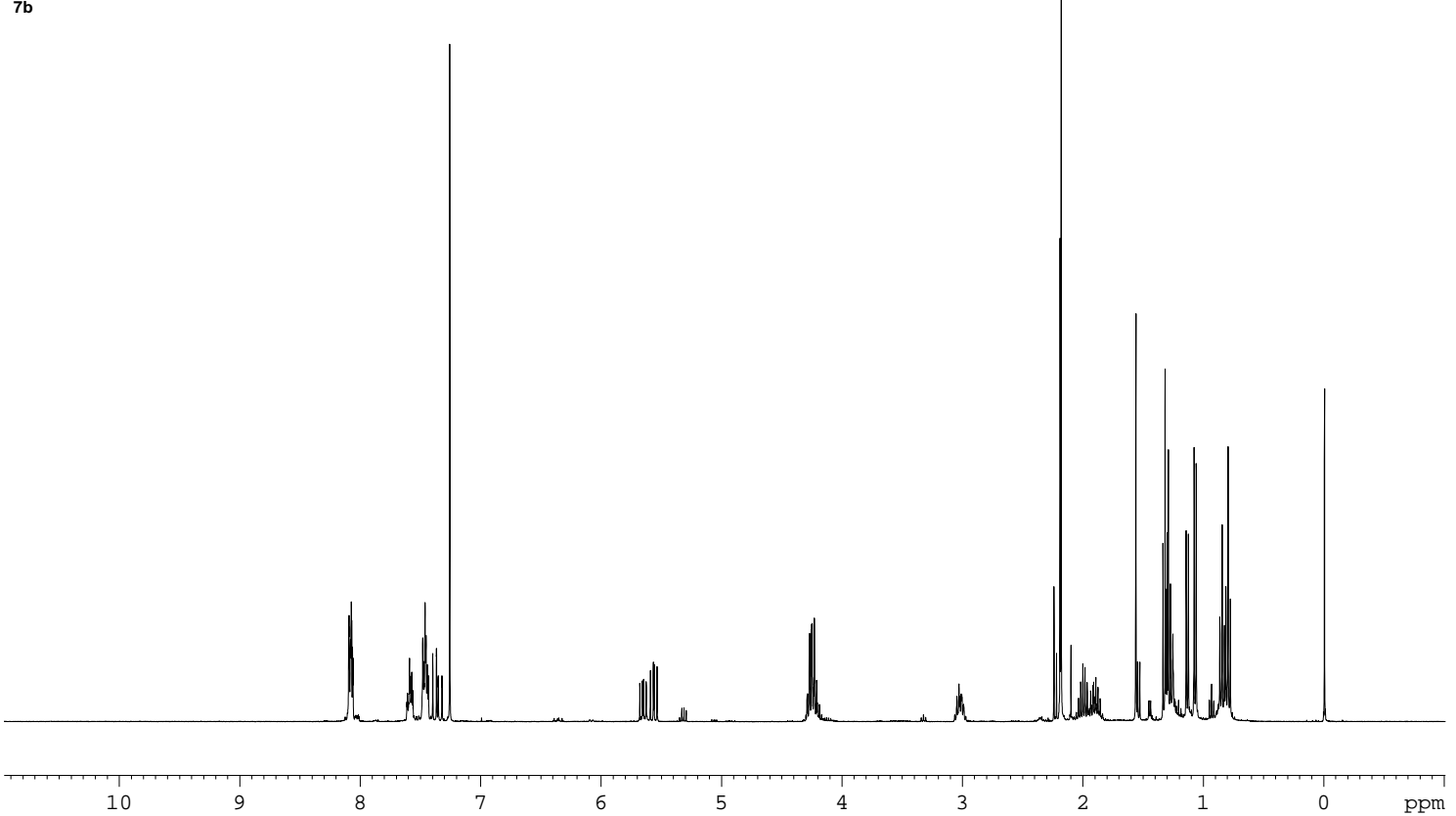

$\mathrm{EtO}_{2} \mathrm{C} \times{ }^{\mathrm{Mt}} \sim \mathrm{OBz}$

$7 b$

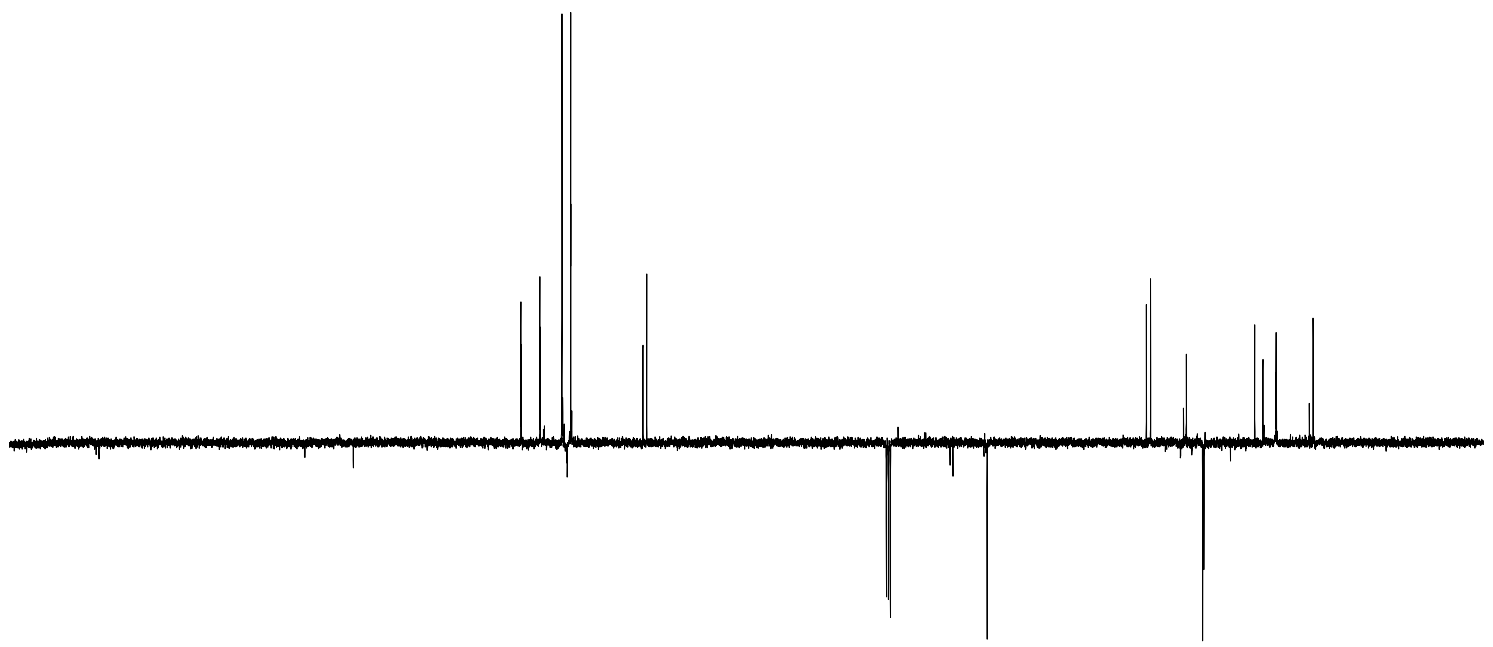

$200 \quad 180$

$160 \quad 140$

100

80

60

40

20

ppm 
MeOc

7c

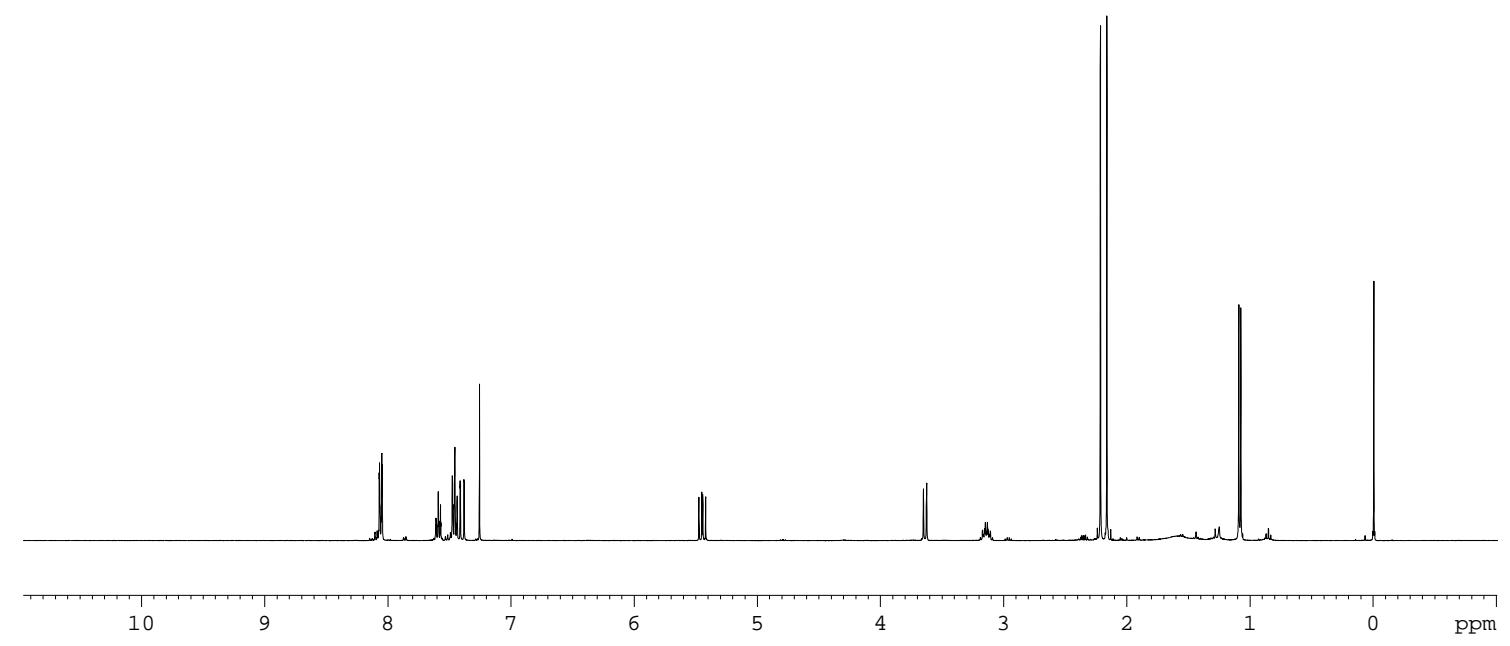

meoc

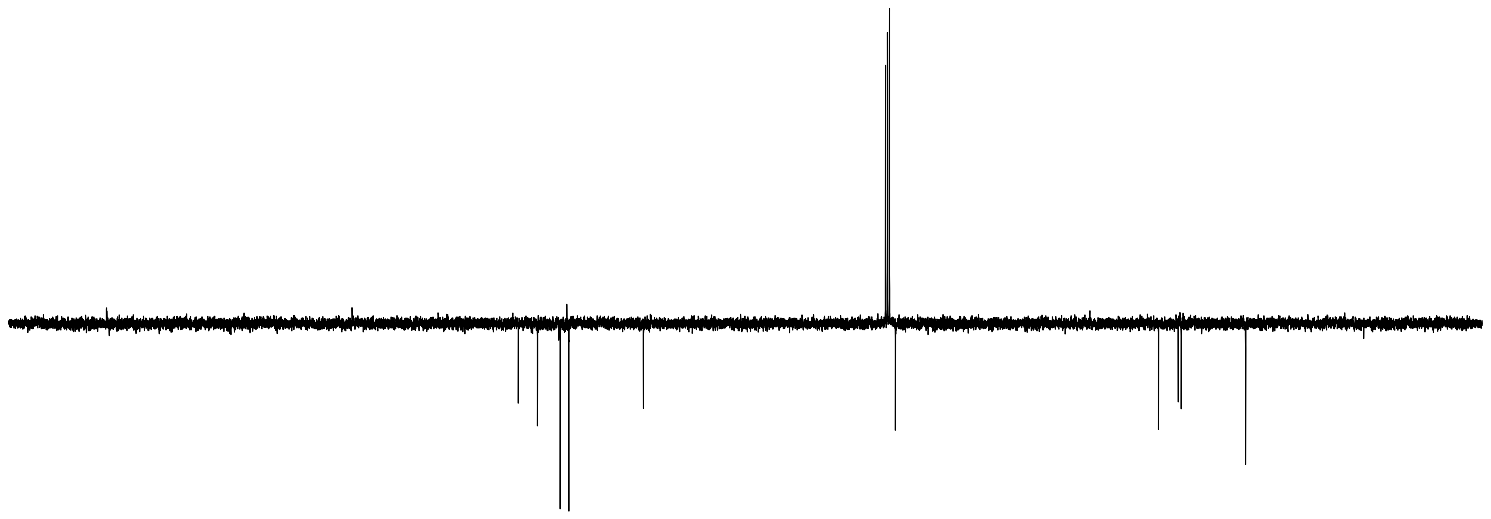

200

180

160

140

120

100

80

60

40

20

ppm 


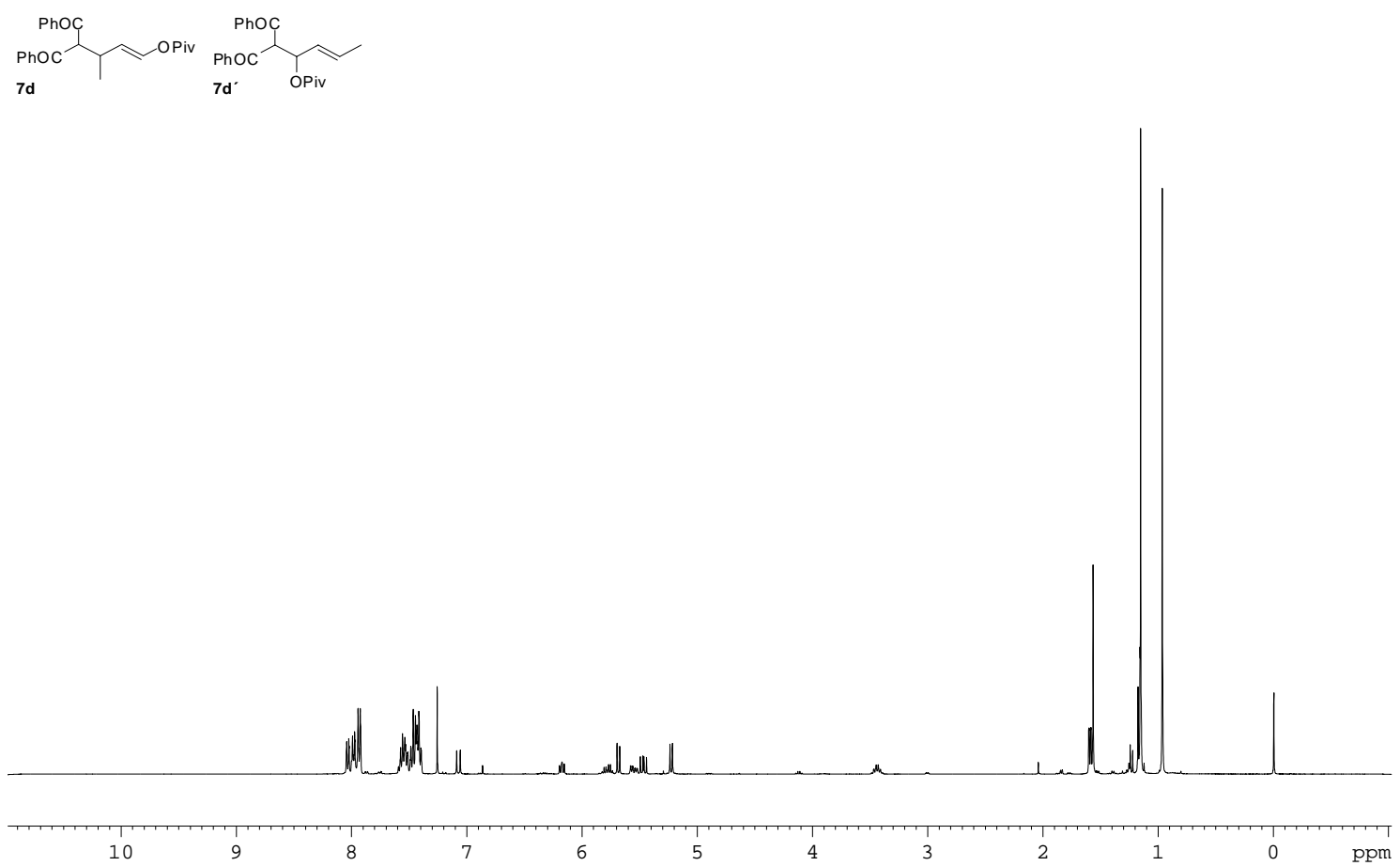

7d

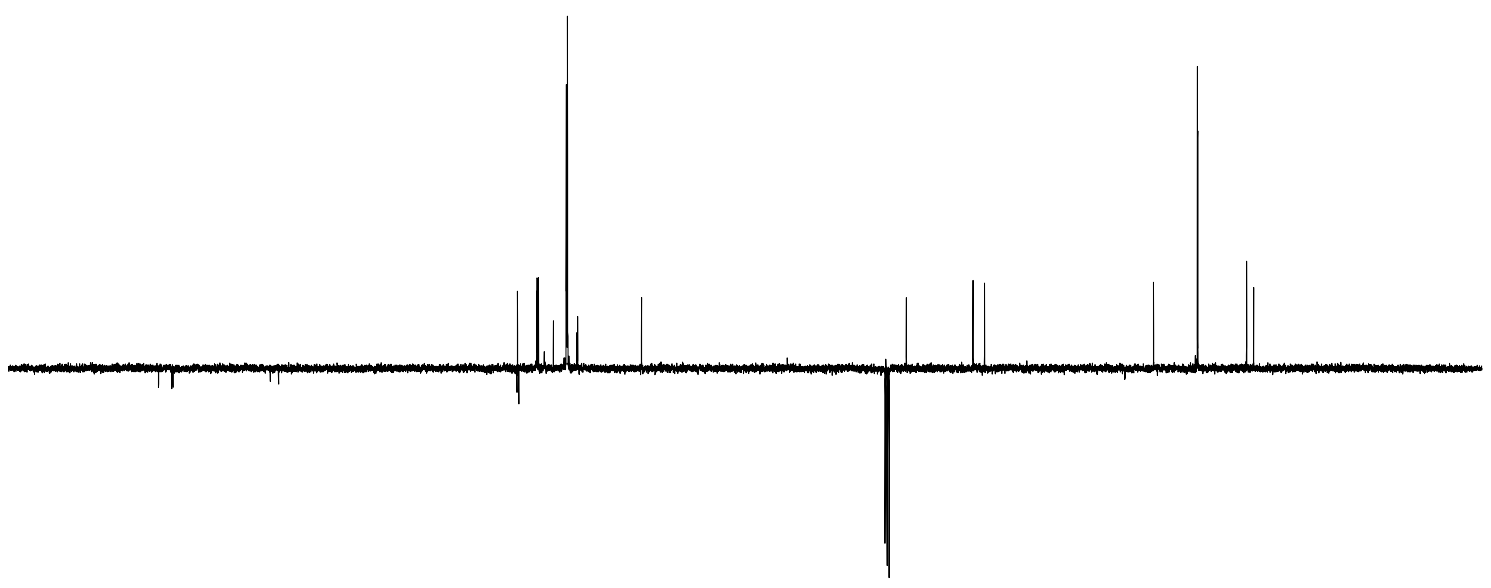

200

180

$160 \quad 140 \quad 120 \quad 100$

$100 \quad 80$

60

40

20

ppm 
n-BuOC

$\mathrm{MeO}_{2} \mathrm{C} \curvearrowright \mathrm{OPiv}$

$7 \mathrm{e}$

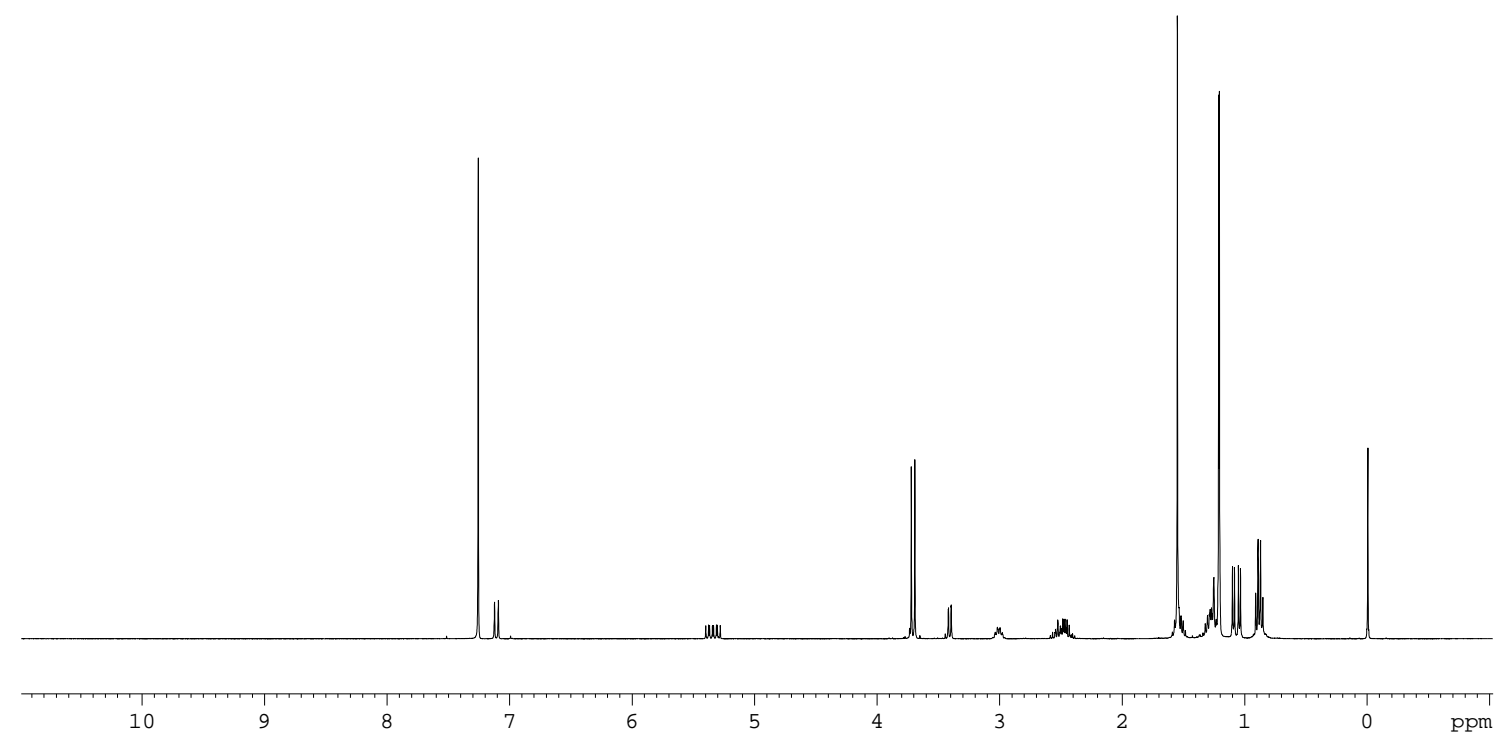

MePiv

$7 e$

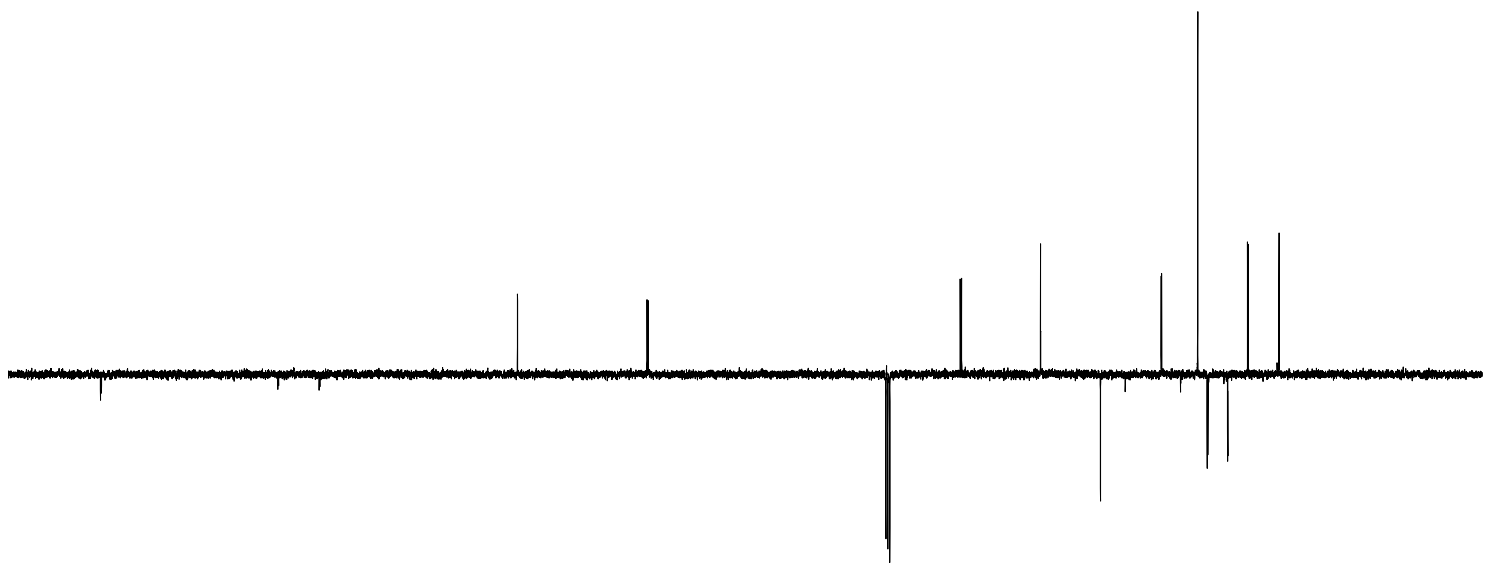

200

$160 \quad 140 \quad 120$

100

80

60

40

20

$\odot \quad \mathrm{ppm}$ 
7f

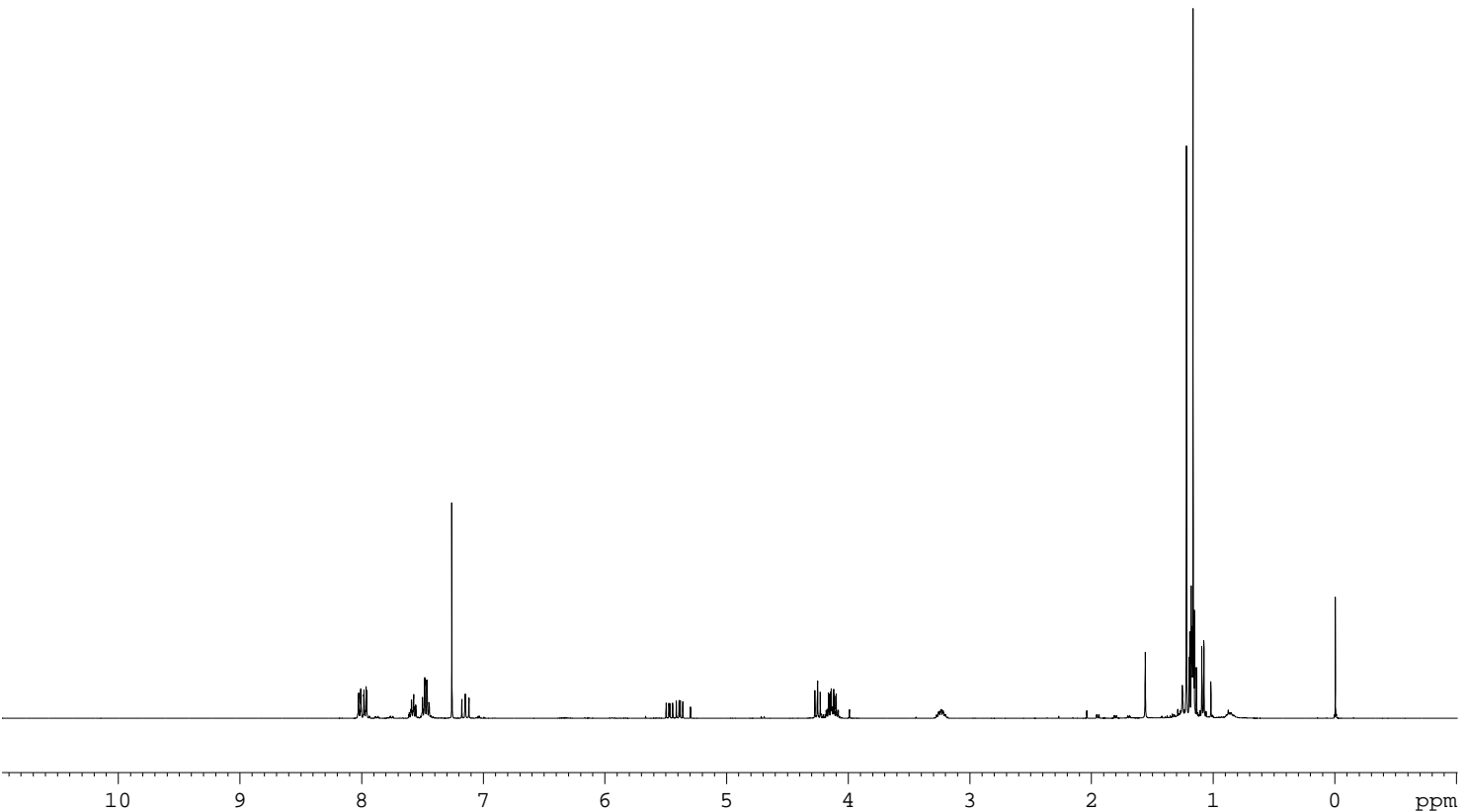

—opiv

$7 f$

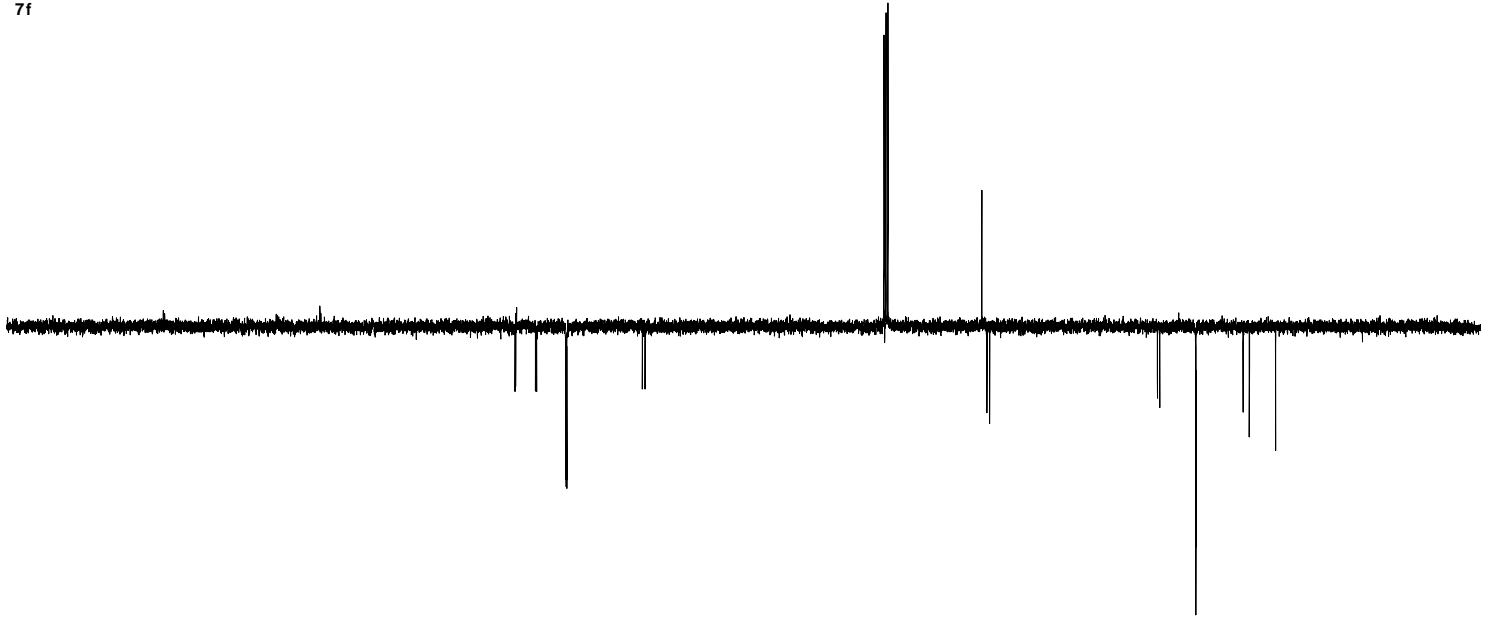

200

180

160

140

120

100

80

60

40

20

ppm 
PhOc $\underbrace{\mathrm{PhOc}}_{7 \mathrm{~g}} \mathrm{OAC}$

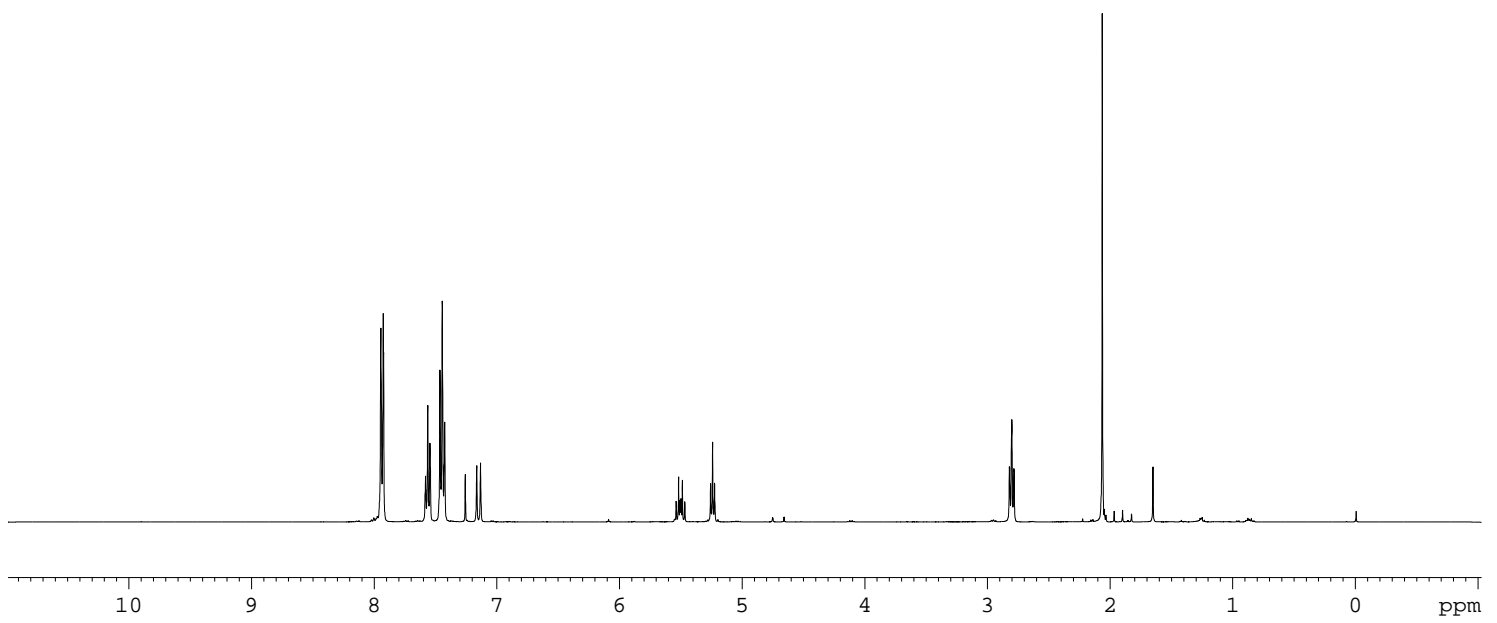

PhOc $\underbrace{\mathrm{PhOC}}_{7 \mathrm{~g}} \mathrm{OAC}$

200

180

160

$140 \quad 120$

100

80

60

40

20 
$\underbrace{\mathrm{D}}_{\mathbf{7 g} \cdot \boldsymbol{d}_{1}}$

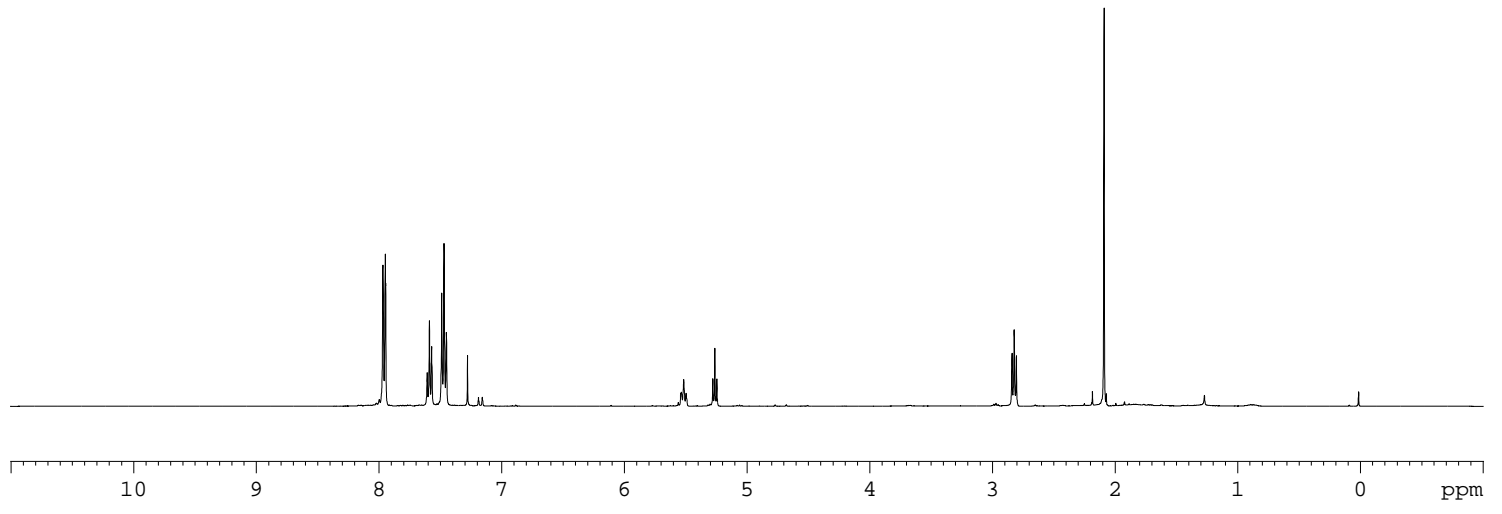

$\underbrace{\mathrm{D}}_{\mathbf{7 g - d _ { 1 }}}$

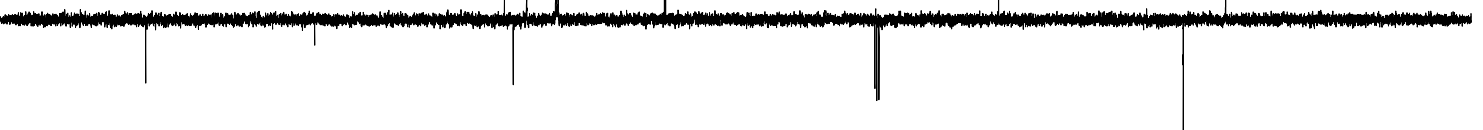

200

180

160

$140 \quad 120$

100

80

60

$4 \odot$

20

ppm 
${\mathbf{7 g} \cdot \boldsymbol{d}_{\mathbf{2}}}_{\substack{\mathrm{D} \\ 80 \%}}^{\mathrm{PhOC}} \mathrm{OAC}$

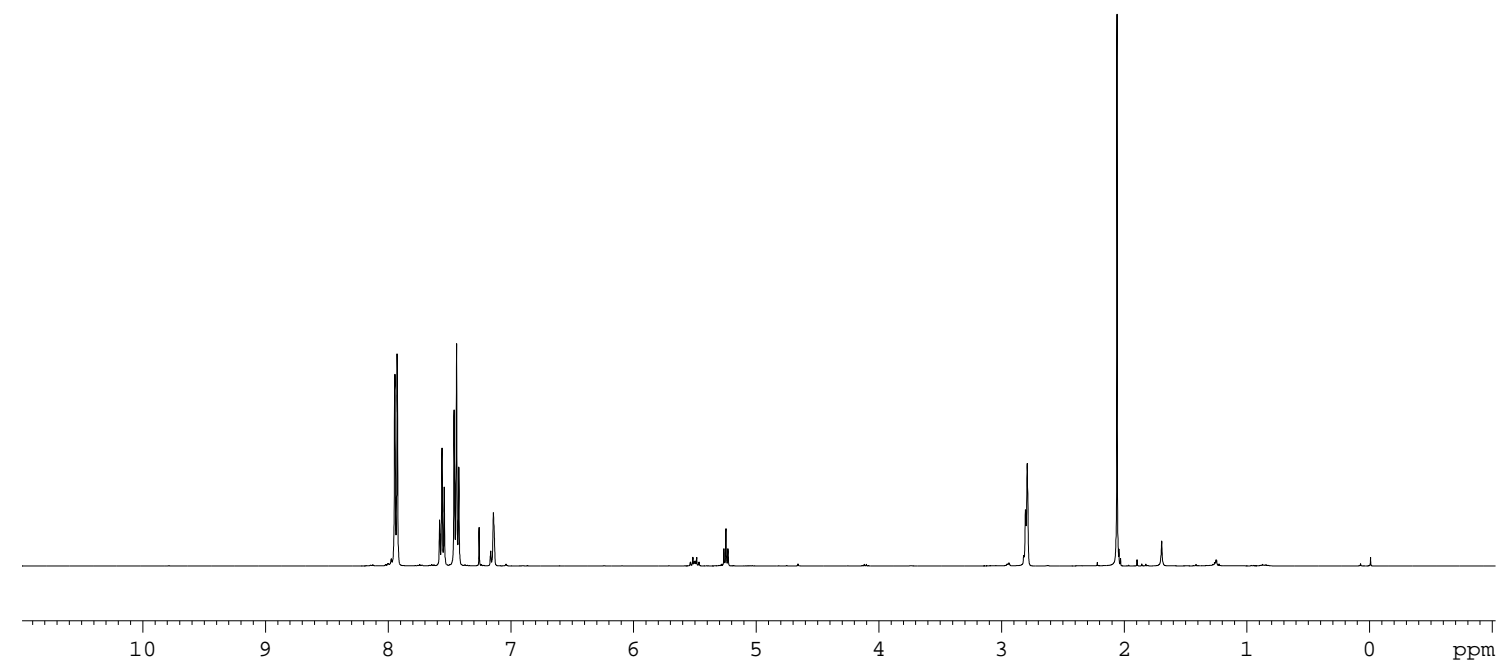

Phoc D, 60\%

Phoc $\underset{D}{\mathrm{D}}$ OAC

$7 g \cdot d_{2}$

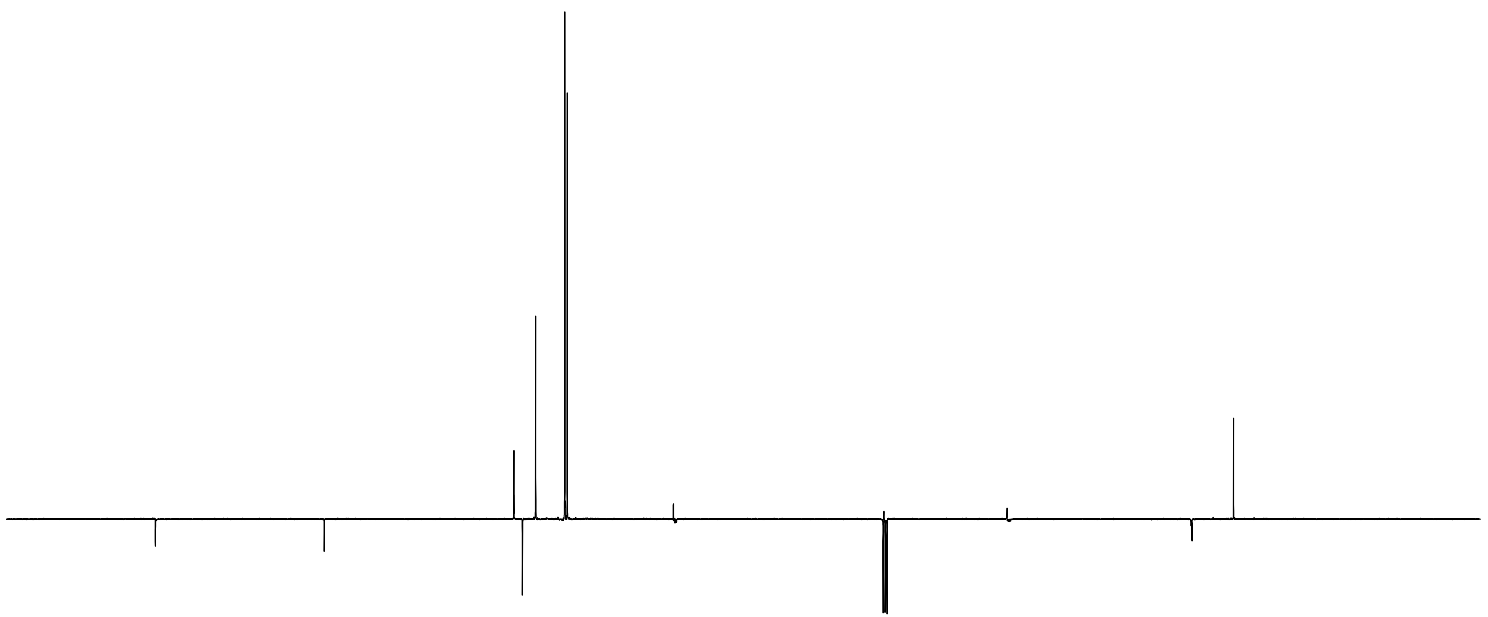

$200 \quad 180$

160

140

120

100

80

$60 \quad 40$

40

20 ppm 

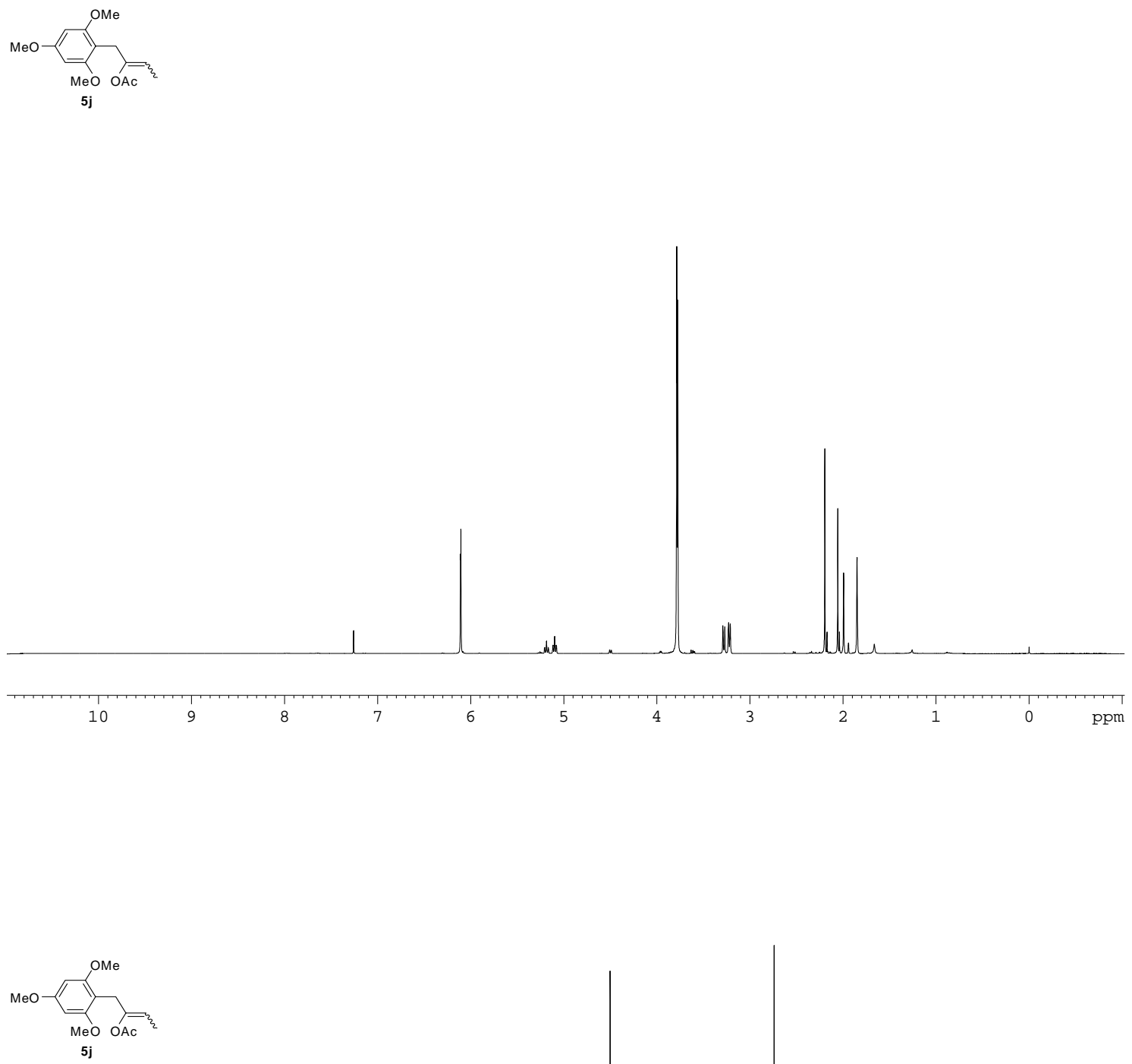

200

160

100

80

60

40

20

$\odot \quad$ ppm 

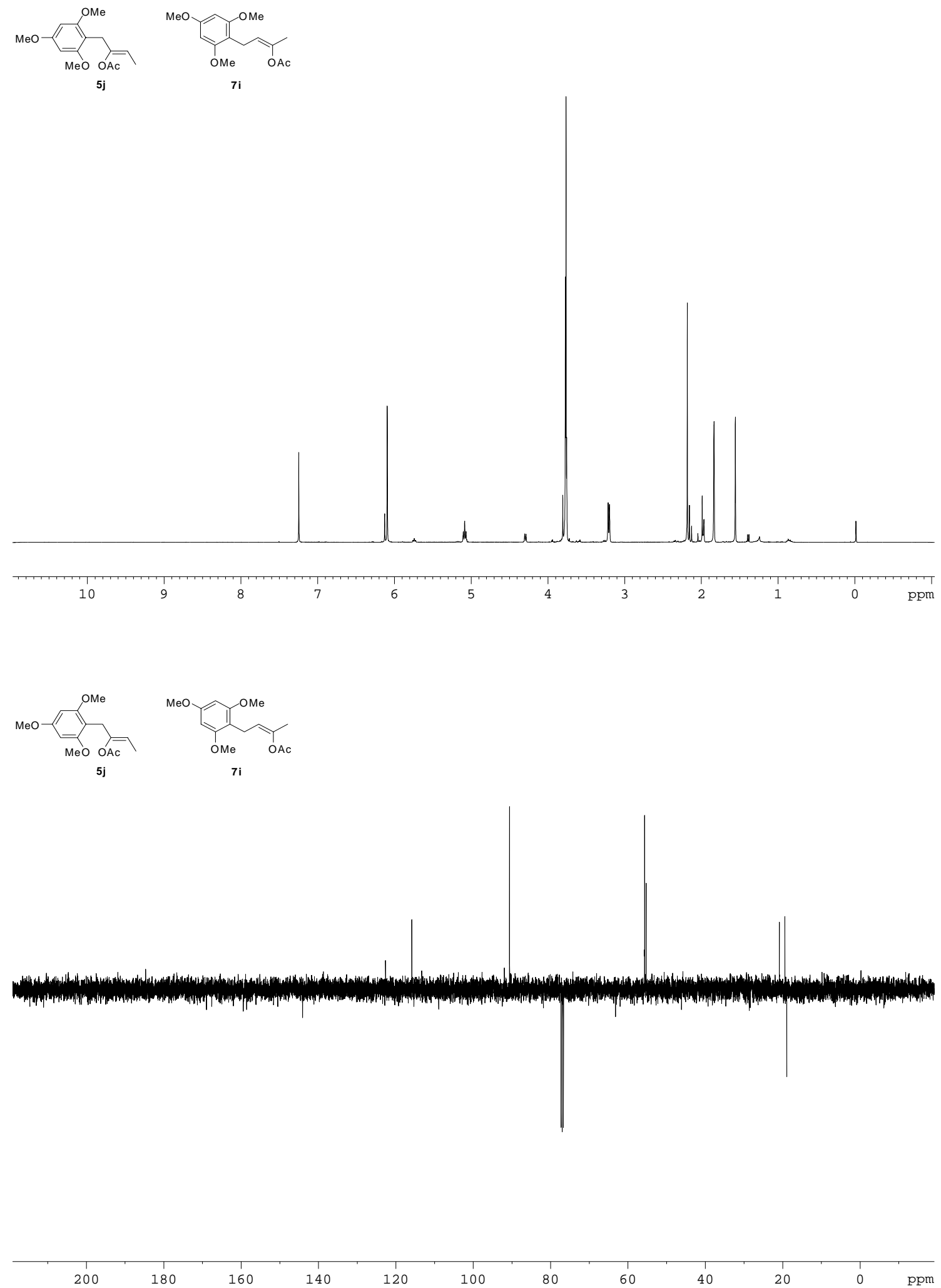
$\overbrace{17}^{0}$

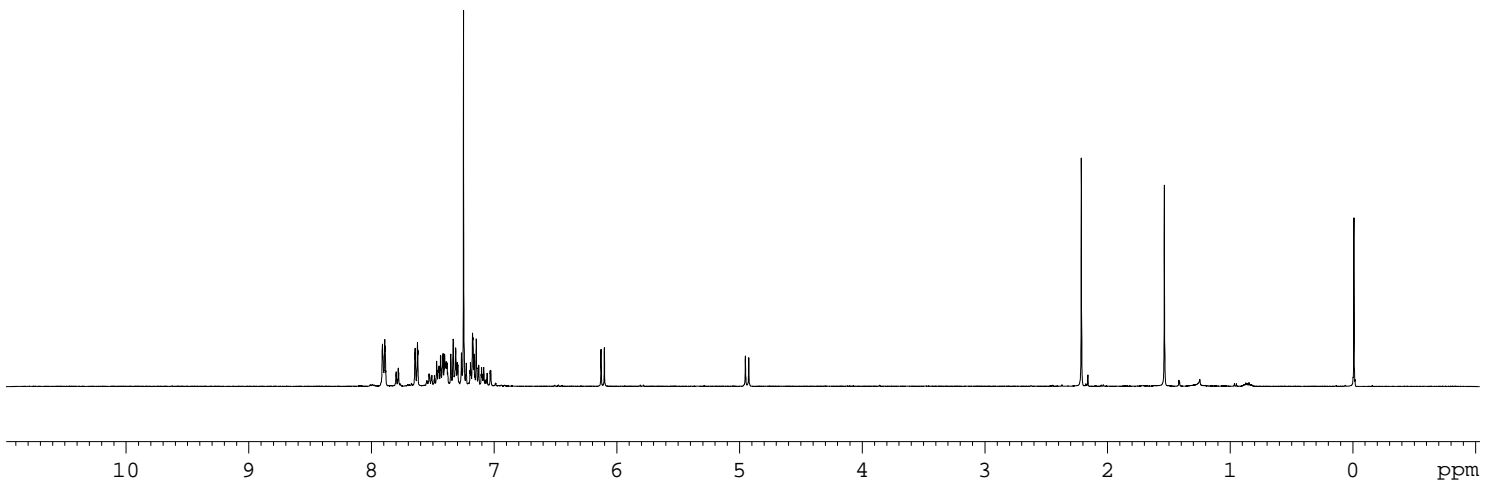

$\underbrace{0}_{17}$

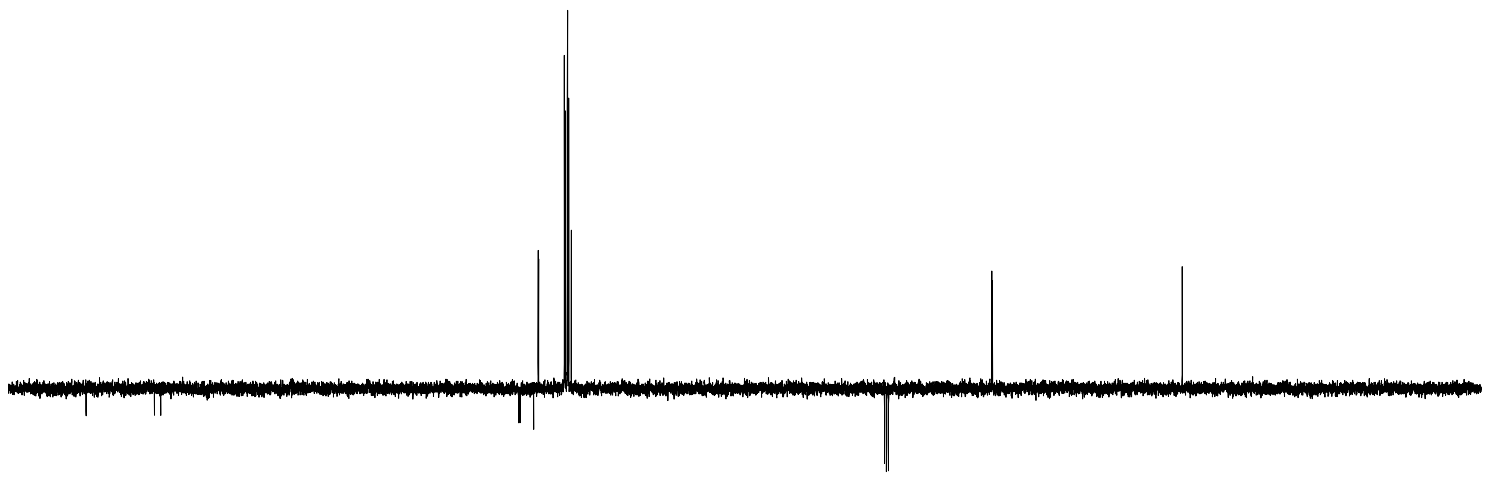

